\title{
Reconstruction of shapes and refractive indices from backscattering experimental data using the adaptivity
}

\author{
Larisa Beilina ${ }^{\circ}$, Nguyen Trung Thành ${ }^{\diamond}$, Michael V. Klibanov ${ }^{\diamond *}$ \\ and John Bondestam Malmberg ${ }^{\circ}$ \\ ${ }^{\circ}$ Department of Mathematical Sciences, \\ Chalmers University of Technology and Gothenburg University, Gothenburg, Sweden \\ Emails: larisa@chalmers.se, john.bondestam.malmberg@chalmers.se. \\ ${ }^{\diamond}$ Department of Mathematics \& Statistics, University of North Carolina at Charlotte, \\ Charlotte, NC, USA. Emails: tnguy152@uncc.edu, mklibanv@uncc.edu
}

\begin{abstract}
We consider the inverse problem of the reconstruction of the spatially distributed dielectric constant $\varepsilon_{r}(\mathbf{x}), \mathbf{x} \in \mathbb{R}^{3}$, which is an unknown coefficient in the Maxwell's equations, from time-dependent backscattering experimental radar data associated with a single source of electric pulses. The refractive index is $n(\mathbf{x})=\sqrt{\varepsilon_{r}(\mathbf{x})}$. The coefficient $\varepsilon_{r}(\mathbf{x})$ is reconstructed using a two-stage reconstruction procedure. In the first stage an approximately globally convergent method proposed is applied to get a good first approximation of the exact solution. In the second stage a locally convergent adaptive finite element method is applied, taking the solution of the first stage as the starting point of the minimization of the Tikhonov functional. This functional is minimized on a sequence of locally refined meshes. It is shown here that all three components of interest of targets can be simultaneously accurately imaged: refractive indices, shapes and locations.
\end{abstract}

Keywords: Coefficient inverse problem, finite element method, globally convergent method, experimental backscattered data.

AMS classification codes: $65 \mathrm{~N} 15,65 \mathrm{~N} 30,35 \mathrm{~J} 25$.

\section{Introduction}

In this paper we investigate the problem of imaging objects placed in air from time-dependent backscattering radar measurements, using a two-stage reconstruction method. In the first stage, initial images are calculated using the globally convergent method for Coefficient Inverse Problems (CIPs), which was originated in 4 with a number of follow up publications; results were summarized in the book 6. In the second stage, those images are refined using an adaptive finite element method (adaptivity) of [10. Results of the first stage for the data sets considered in this paper were presented in $[8,26$. Here, we present the results of the second stage. Only the maximum value of the dielectric constant and the location of a target were accurately reconstructed in $[8,26$, using the globally convergent method. The accuracy of the reconstruction of the shape of the target was limited. Using the two-stage reconstruction procedure, it is shown here that we can simultaneously and accurately reconstruct all three components of interest of objects: refractive indices, shapes, and locations.

We reconstruct these three components simultaneously as parts of an unknown coefficient, which is the spatially varying dielectric constant $\varepsilon_{r}(\mathbf{x}), \mathbf{x} \in \mathbb{R}^{3}$, in the Maxwell's equations. Below $\mathbf{x}=(x, y, z) \in \mathbb{R}^{3}$, where $x$ is the horizontal axis, $y$ is the vertical axis and $z$ is the axis which points from the target

*Corresponding author 


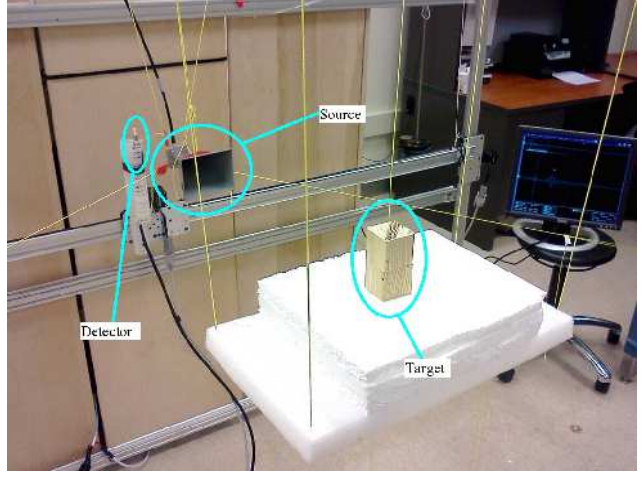

(a)

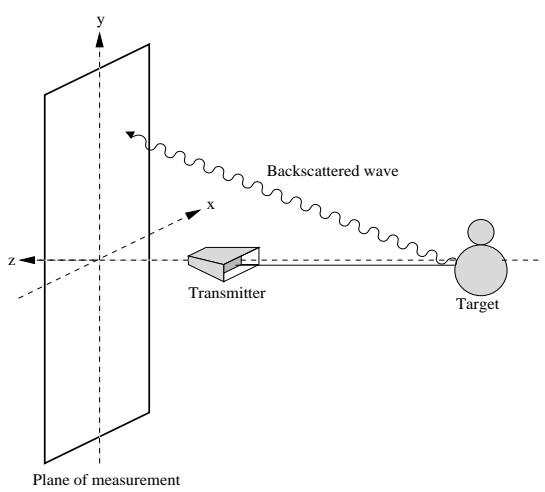

(b)

Figure 1: (a): Experimental setup; (b) Our data collection scheme.

towards the measurement plane, see Figure 1 Even though only one component $E_{2}$ of the electric field $E=\left(E_{1}, E_{2}, E_{3}\right)$ was measured by our experimental device, we numerically solve here a CIP for the three-dimensional (3-d) Maxwell's equations. The boundary data for two other components $E_{1}, E_{3}$ are obtained via computational simulations.

Experimental data were collected by a microwave device which was recently assembled at the University of North Carolina at Charlotte, USA. Our desired application is imaging of explosives. In this paper we consider only targets located in air. The work on real data for the case when targets are buried under the ground is reported in [27. Note that explosives may be located in air [16], e.g., improvised explosive devices (IEDs). We image both homogeneous and heterogeneous targets. Heterogeneous targets model IEDs.

To collect those data, a single location of the source of electric pulses was used. Hence, we used the minimal amount of the information. The use of more sources was both hard to arrange experimentally and undesirable for our target application. The backscattering time dependent signal was measured at a number of detectors covering a part of a plane, i.e., over a narrow range of backscattering angles, see Figure 1. That plane was placed behind the source. Experimental data of this paper were collected for targets located in air on the distance of 80 centimeters from the measurement plane, which is 20 wavelengths, i.e., in a far field zone. The distance between neighboring detectors was 2 centimeters.

We refer to, e.g., [18, 19] for treatments of experimental data in the frequency domain by other numerical methods for CIPs for Maxwell's equations. In particular, blind real data were considered in [19. As to the adaptivity technique for inverse problems, we refer to, e.g., [11, 20]. There are many works dedicated to inverse problems of shape reconstruction; we refer to some most recent ones, e.g., 21, 22, 28, 29. We also refer to [14] for a survey about inverse problems of shape reconstruction.

An outline of this paper is as follows. In section 2 we describe the two-stage reconstruction procedure. In section 3 we state the forward and inverse problems. In section 4 we present Tikhonov functional and optimality conditions. In section 5 we describe the finite element method used in our computation. In section 6 we present the mesh refinement recommendation and the adaptive algorithm. Some details of numerical implementation are described in section 7 In section 8 we present reconstruction results. Finally, a summary is given in section 9 .

\section{Two-stage reconstruction procedure}

In [8, 26] we have considered the problem of the reconstruction of the spatially distributed dielectric constant $\varepsilon_{r}(\mathbf{x}), \mathbf{x} \in \mathbb{R}^{3}$ from experimental data, which were the same as in the current paper. In [8, 26] 
this function was the unknown coefficient in a wave-like PDE

$$
\varepsilon_{r}(\mathbf{x}) \frac{\partial^{2} E_{2}}{\partial t^{2}}=\Delta E_{2}
$$

To reconstruct $\varepsilon_{r}(\mathbf{x})$, we have used the approximately globally convergent algorithm of [6]. The notion of the approximate global convergence ("global convergence" in short) was introduced in [6, 7]. Indeed, conventional least squares cost functionals for CIPs are non convex and typically have many local minima. Hence, given a CIP, the first question to address in its numerical treatment is: How to obtain a good approximation for the exact solution without any a priori knowledge of a small neighborhood of this solution? We call a numerical method addressing this question globally convergent.

It is well known that it is tremendously difficult to address this question. For this reason, a certain reasonable approximation was made in 6, 7. This approximation is used only on the first iteration of that method. Because of this approximation, we call the technique of [6, 7] approximately globally convergent. Due to that approximation, a room is left for a refinement of results.

An important point here is that there exists a rigorous guarantee within the framework of that approximate model that the solution resulting from the globally convergent method is located in a small neighborhood of the exact solution. This is achieved without any a priori knowledge of a small neighborhood of the exact solution. Thus, a locally convergent numerical method can be used for a refinement of the solution obtained by the globally convergent technique of [6, 7].

The latter is the main goal of the current paper. We synthesize here the adaptive finite element method of [10] with the globally convergent numerical method of [6] in order to improve the reconstruction of shapes of objects imaged in [8, 26]. The idea of this synthesis was first introduced in [5]. The synthesis represents the following two-stage reconstruction procedure:

Stage 1. In this stage the approximately globally convergent method of [6] is applied and a good first approximation for the exact solution is obtained.

Stage 2. This stage refines the solution obtained in the first stage. The locally convergent adaptivity technique of [10] is applied. The solution obtained in [8, 26] in the first stage is taken as the starting point in the minimization procedure of the Tikhonov functional.

An important advantage of using the two-stage reconstruction procedure follows from Theorem 1.9.1.2 of [6]. This theorem states that the minimizer of the Tikhonov functional (i.e., the regularized solution) is closer to the exact solution than the first guess, provided, however, that the first guess is sufficiently close to the exact solution. Therefore, that first guess should be delivered by the globally convergent method of the first stage. The adaptive finite element method of [10], which we use in the second stage, minimizes the Tikhonov functional on a sequence of locally refined meshes, which is the main attractive point of the adaptivity. It enables one to maintain a reasonable compromise between not using an exceedingly large number of finite elements and a good accuracy of the resulting solution. It follows from Theorem 4.9.3 of [6] and Theorem 5.2 of [3] that if the first guess is sufficiently close to the exact solution, then the accuracy of its reconstruction monotonically improves with local mesh refinements. On the other hand, it was shown in section 5.8.4 of the book [6] that a locally convergent numerical method taken alone does not work for transmitted time dependent experimental data generated by a single source. The same conclusion was drawn for a different type of experimental data in [23].

\section{Statement of Forward and Inverse Problems}

We model the electromagnetic wave propagation in an isotropic, non-magnetic space $\mathbb{R}^{3}$ with the dielectric constant coefficient $\varepsilon_{r}(\mathbf{x})$. The electric field $E=\left(E_{1}, E_{2}, E_{3}\right)$ satisfies the following Cauchy problem:

$$
\begin{aligned}
\varepsilon_{r}(\mathbf{x}) \frac{\partial^{2} E}{\partial t^{2}}+\nabla \times(\nabla \times E) & =\left(0, \delta\left(z-z_{0}\right) f(t), 0\right), \text { in } \mathbb{R}^{3} \times(0, T), \\
E(\mathbf{x}, 0)=0, \quad E_{t}(\mathbf{x}, 0) & =0 \text { in } \mathbb{R}^{3} .
\end{aligned}
$$

where $f(t) \not \equiv 0$ is the time-dependent waveform of the component $E_{2}$ of the incident plane wave, which is originated at the plane $\left\{z=z_{0}\right\}$ and propagates along the $z$-axis. In our experiment the component 
$E_{2}$ corresponds to the electromagnetic pulse which is sent into the medium. Thus, in (2) as well as in our computer simulations of section 7 the incident field has only one non-zero component $E_{2}(\mathbf{x}, t)$. This component propagates along the $z$-axis until it reaches the target, where it is scattered.

Let $\Omega \subset \mathbb{R}^{3}$ be a convex bounded domain. We impose the following conditions on the coefficient $\varepsilon_{r}(\mathbf{x})$ :

$$
\varepsilon_{r}(\mathbf{x}) \in C^{\alpha}\left(\mathbb{R}^{3}\right), \varepsilon_{r}(\mathbf{x}) \in[1, d], \quad \varepsilon_{r}(\mathbf{x})=1 \text { for } \mathbf{x} \in \mathbb{R}^{3} \backslash \Omega,
$$

where $d=$ const. $>1$. We a priori assume the knowledge of the constant $d$. This means the knowledge of the set of admissible coefficients in (3). However, we do not impose small-value assumptions on the unknown coefficient $\varepsilon_{r}(\mathbf{x})$, i.e., we do not assume that $d$ is small. Here $C^{\alpha}, \alpha \in(0,1)$, is the Hölder space. Let $\Gamma \subset \partial \Omega$ be the part of the boundary $\partial \Omega$ on which the backscattered data are measured.

Coefficient Inverse Problem (CIP): Suppose that the coefficient $\varepsilon_{r}(\mathbf{x})$ satisfies conditions (3) and that $\bar{\Omega} \cap\left\{z=z_{0}\right\}=\varnothing$. Determine the function $\varepsilon_{r}(\mathbf{x})$ for $\mathbf{x} \in \Omega$, assuming that the following function $g(\mathbf{x}, t)$ is known for a single incident plane wave

$$
g(\mathbf{x}, t)=E(\mathbf{x}, t), \forall(\mathbf{x}, t) \in \Gamma \times(0, \infty)
$$

\subsection{Domain decomposition finite element/finite difference method}

It is impossible to solve the problem (2) in the whole space $\mathbb{R}^{3}$. Hence, we solve it in a bounded domain $G$ which contains our domain of interest $\Omega$. For the convenience of our local mesh refinement procedure, we use the domain decomposition finite element/finite difference method of [9. For this purpose, we decompose $G$ as $G=\Omega_{F E M} \cup \Omega_{F D M}$ with $\Omega_{F E M}=\Omega$. Here we use a finite element mesh in $\Omega_{F E M}$, and in $\Omega_{F D M}$ we use a finite difference mesh. These two domains have a thin layer of structured overlapping nodes where we use an exchange procedure between computational solutions obtained by finite element and finite difference methods, see details in 9 . By (3)

$$
\begin{aligned}
& \varepsilon_{r}(\mathbf{x}) \geq 1, \text { for } \mathbf{x} \in \Omega_{F E M}, \\
& \varepsilon_{r}(\mathbf{x})=1, \text { for } \mathbf{x} \in \Omega_{F D M} .
\end{aligned}
$$

In our computation we use the following model problem in the computational domain $G$ for the electric field $E$ with the stabilizing divergence condition [1] with $s \geq 1$ and with boundary conditions specified in this section below:

$$
\begin{aligned}
\varepsilon_{r} \frac{\partial^{2} E}{\partial t^{2}}+\nabla \times(\nabla \times E)-s \nabla\left(\nabla \cdot\left(\varepsilon_{r} E\right)\right) & =0, \text { in } G \times(0, T), \\
E(\mathbf{x}, 0)=0, \quad E_{t}(\mathbf{x}, 0) & =0 \text { in } G .
\end{aligned}
$$

For simplicity, we choose the domains $\Omega$ and $G$ by

$$
\begin{gathered}
\Omega=\Omega_{F E M}=\left\{\mathbf{x}=(x, y, z):-a<x<a,-b<y<b,-c<z<c_{1}\right\}, \\
G=\left\{\mathbf{x}=(x, y, z):-X<x<X,-Y<y<Y,-Z<z<z_{0}\right\},
\end{gathered}
$$

with positive numbers $a, b, c, X>a, Y>b,-Z<-c<c_{1}<z_{0}$ and $\Omega_{F D M}=G \backslash \Omega_{F E M}$. Denote by

$$
\partial_{1} G:=\bar{G} \cap\left\{z=z_{0}\right\}, \quad \partial_{2} G:=\bar{G} \cap\{z=-Z\}, \quad \partial_{3} G:=\partial G \backslash\left(\partial_{1} G \cup \partial_{2} G\right) .
$$

The backscattering side of $\Omega$ is $\Gamma=\partial \Omega \cap\left\{z=c_{1}\right\}$. Next, define $\partial_{i} G_{T}:=\partial_{i} G \times(0, T), i=1,2,3$. Let $t_{1} \in(0, T)$ be a number, and we assume that the function $f(t) \in C\left[0, t_{1}\right]$ and $f(t)=0$, for $t>t_{1}$. We impose the following boundary conditions

$$
\begin{gathered}
E(\mathbf{x}, t)=(0, f(t), 0) \text { on } \partial_{1} G \times\left(0, t_{1}\right], \\
\partial_{n} E(\mathbf{x}, t)=-\partial_{t} E(\mathbf{x}, t) \text { on } \partial_{1} G \times\left(t_{1}, T\right),
\end{gathered}
$$




$$
\begin{gathered}
\partial_{n} E(\mathbf{x}, t)=-\partial_{t} E(\mathbf{x}, t) \text { on } \partial_{2} G_{T}, \\
\partial_{n} E(\mathbf{x}, t)=0 \text { on } \partial_{3} G_{T},
\end{gathered}
$$

where $\partial_{n}$ is the normal derivative. Conditions (12), (13) are first order absorbing boundary conditions 13 at the planes $\partial_{1} G$ and $\partial_{2} G$ of the rectangular prism $G$, and (14) is the zero Neumann condition at the lateral part $\partial_{3} G$ of the boundary $\partial G$. Condition (11) means that the incident plane wave is emitted only up to the time $t=t_{1}$ and then propagates inside of the domain $G$.

It was demonstrated numerically in [9] that the solution of the problem (6)-(14) approximates well the solution of the original Maxwell's equations for $s=1$. The energy estimate of Theorem 4.1 of [9] guarantees the stability of the forward problem (6)-(14) for $s \geq 1$.

Using the transformation $\nabla \times(\nabla \times E)=\nabla(\nabla \cdot E)-\nabla \cdot(\nabla E)$, the model problem (6), (7), (11) (14) can be rewritten as

$$
\begin{aligned}
\varepsilon_{r} \frac{\partial^{2} E}{\partial t^{2}}+\nabla(\nabla \cdot E)-\nabla \cdot(\nabla E)-s \nabla\left(\nabla \cdot\left(\varepsilon_{r} E\right)\right) & =0, \text { in } G \times(0, T), \\
E(\mathbf{x}, 0)=0, \quad E_{t}(\mathbf{x}, 0) & =0 \text { in } G, \\
E(\mathbf{x}, t) & =(0, f(t), 0) \text { on } \partial_{1} G \times\left(0, t_{1}\right], \\
\partial_{n} E(\mathbf{x}, t) & =-\partial_{t} E(\mathbf{x}, t) \text { on } \partial_{1} G \times\left(t_{1}, T\right), \\
\partial_{n} E(\mathbf{x}, t) & =-\partial_{t} E(\mathbf{x}, t) \text { on } \partial_{2} G_{T}, \\
\partial_{n} E(\mathbf{x}, t) & =0 \text { on } \partial_{3} G_{T} .
\end{aligned}
$$

We refer to 9 for details of the numerical solution of the forward problem (15)-(20).

\section{Tikhonov functional and optimality conditions}

Let $\Gamma_{1}$ be the extension of the backscattering side $\Gamma$ up to the boundary $\partial_{3} G$ of the domain $G$, i.e.,

$$
\Gamma_{1}=\left\{\mathbf{x}=(x, y, z):-X<x<X,-Y<y<Y, z=c_{1}\right\} .
$$

Let $G_{b}$ be the part of the rectangular prism $G$ which lies between the two planes $\Gamma_{1}$ and $\{z=-Z\}$ :

$$
G_{b}=\left\{\mathbf{x}=(x, y, z):-X<x<X,-Y<y<Y,-Z<z<c_{1}\right\} .
$$

Denote by $Q_{T}=G_{b} \times(0, T), S_{T}=\partial G_{b} \times(0, T)$. Even though we have the data $g(\mathbf{x}, t)$ in (4) only on $\Gamma$, we show in subsection 7.3 .3 below how we complement these data on the rest of the boundary $\partial G_{b}$ of the domain $G_{b}$, i.e., on $\partial G_{b} \backslash \Gamma$. This way we approximately obtain the function $\widetilde{g}(\mathbf{x}, t)$ :

$$
\widetilde{g}(\mathbf{x}, t)=E(\mathbf{x}, t), \forall(\mathbf{x}, t) \in S_{T} .
$$

We reformulate our inverse problem as an optimization problem. Thus, we find $\varepsilon_{r}$ by minimizing the Tikhonov functional:

$$
F\left(E, \varepsilon_{r}\right):=\frac{1}{2} \int_{S_{T}}(E-\tilde{g})^{2} z_{\delta}(t) d \mathbf{x} d t+\frac{1}{2} \gamma \int_{G}\left(\varepsilon_{r}-\varepsilon_{r g l o b}\right)^{2} d \mathbf{x}
$$

where $\gamma>0$ is the regularization parameter, and $\varepsilon_{r, g l o b}(\mathbf{x})$ is the computed coefficient via the globally convergent method.

Let $E_{\text {glob }}(\mathbf{x}, t)$ be the solution of the forward problem (15)-(20) with $\varepsilon_{r}(\mathbf{x}):=\varepsilon_{r, g l o b}(\mathbf{x})$. Denote by $p(\mathbf{x}, t)=\left.\partial_{n} E_{g l o b}(\mathbf{x}, t)\right|_{S_{T}}$. In addition to the Dirichlet condition (23), we set the Neumann boundary condition as

$$
\partial_{n} E(\mathbf{x}, t)=p(\mathbf{x}, t), \forall(\mathbf{x}, t) \in S_{T} .
$$


To formulate the Fréchet derivative of the Tikhonov functional (24) (see formula (36) below), we make use of the adjoint method. This method is based on the state and adjoint problems. The state problem in the domain $G_{b}$ is given by

$$
\begin{aligned}
\varepsilon_{r} \frac{\partial^{2} E}{\partial t^{2}}+\nabla(\nabla \cdot E)-\nabla \cdot(\nabla E)-s \nabla\left(\nabla \cdot\left(\varepsilon_{r} E\right)\right) & =0, \text { in } Q_{T}, \\
E(\mathbf{x}, 0)=0, \quad E_{t}(\mathbf{x}, 0) & =0 \text { in } G_{b}, \\
\partial_{n} E(\mathbf{x}, t) & =p(\mathbf{x}, t) \text { on } S_{T} .
\end{aligned}
$$

The adjoint problem is:

$$
\begin{aligned}
\varepsilon_{r} \frac{\partial^{2} \lambda}{\partial t^{2}}+\nabla(\nabla \cdot \lambda)-\nabla \cdot(\nabla \lambda)-s \varepsilon_{r} \nabla(\nabla \cdot \lambda) & =0, \text { in } Q_{T}, \\
\lambda(\mathbf{x}, T)=0, \quad \lambda_{t}(\mathbf{x}, T) & =0 \text { in } G_{b}, \\
\partial_{n} \lambda(\mathbf{x}, t) & =z_{\delta}(t)(\widetilde{g}-E)(\mathbf{x}, t) \text { on } S_{T} .
\end{aligned}
$$

Here, $z_{\delta}(t)$ is used to ensure the compatibility conditions at $\bar{Q}_{T} \cap\{t=T\}$ for the adjoint problem and $\delta>0$ is a small number. The function $z_{\delta}(t)$ is chosen such that

$$
z_{\delta} \in C^{\infty}[0, T], z_{\delta}(t)= \begin{cases}1 & \text { for } t \in[0, T-\delta], \\ 0 & \text { for } t \in\left(T-\frac{\delta}{2}, T\right], \\ 0<z_{\delta}<1 & \text { for } t \in\left(T-\delta, T-\frac{\delta}{2}\right),\end{cases}
$$

Weak solutions $E, \lambda \in H^{1}\left(Q_{T}\right)$ to problems (26)-(28) and (29)-(31) are defined similarly with the case of only one hyperbolic equation in Chapter 4 of the book [17, also see formula (34) in 9]. The weak solution to the state problem (26)-(28) is the solution to the following equation:

$$
\begin{aligned}
& \int_{Q_{T}}\left(-\varepsilon_{r} \frac{\partial E}{\partial t} \frac{\partial v}{\partial t} d \mathbf{x} d t-\int_{Q_{T}}(\nabla \cdot E)(\nabla \cdot v) d \mathbf{x} d t+\int_{Q_{T}}(\nabla E)(\nabla v) d \mathbf{x} d t\right. \\
& +s \int_{Q_{T}}\left(\nabla \cdot\left(\varepsilon_{r} E\right)\right)(\nabla \cdot v) d \mathbf{x} d t-\int_{\partial S_{T}} v p d \sigma d t=0, \forall v \in H^{1}\left(Q_{T}\right), v(x, T)=0,
\end{aligned}
$$

The weak solution to the adjoint problem (29)-(31) is the solution to the following equation:

$$
\begin{aligned}
& -\int_{S_{T}}(\tilde{g}-E) w z_{\delta} d \sigma d t-\int_{Q_{T}} \varepsilon_{r} \frac{\partial \lambda}{\partial t} \frac{\partial w}{\partial t} d \mathbf{x} d t-\int_{Q_{T}}(\nabla \cdot \lambda)(\nabla \cdot w) d \mathbf{x} d t \\
& +\int_{Q_{T}}(\nabla \lambda)(\nabla w) d \mathbf{x} d t+s \int_{Q_{T}}(\nabla \cdot \lambda)\left(\nabla \cdot\left(\varepsilon_{r} w\right)\right) d \mathbf{x} d t, \forall w \in H^{1}\left(Q_{T}\right), w(x, 0)=0 .
\end{aligned}
$$

Introduce the following spaces of real valued vector functions

$$
\begin{gathered}
H_{E}^{1}\left(Q_{T}\right)=\left\{f \in\left[H^{1}\left(Q_{T}\right)\right]^{3}: f(\mathbf{x}, 0)=0\right\}, \\
H_{\lambda}^{1}\left(Q_{T}\right)=\left\{f \in\left[H^{1}\left(Q_{T}\right)\right]^{3}: f(\mathbf{x}, T)=0\right\}, \\
U^{1}=H_{E}^{1}\left(G_{T}\right) \times H_{\lambda}^{1}\left(G_{T}\right) \times B(G),
\end{gathered}
$$

where $B(G)$ is the space of functions bounded on $G$ with the norm $\|f\|_{B(G)}=\sup _{G}|f|$. To minimize the functional (24) we introduce the Lagrangian

$$
\begin{aligned}
L\left(E, \lambda, \varepsilon_{r}\right) & =F\left(E, \varepsilon_{r}\right)-\int_{Q_{T}} \varepsilon_{r} \frac{\partial \lambda}{\partial t} \frac{\partial E}{\partial t} d \mathbf{x} d t-\int_{Q_{T}}(\nabla \cdot E)(\nabla \cdot \lambda) d \mathbf{x} d t \\
& +\int_{Q_{T}}(\nabla E)(\nabla \lambda) d \mathbf{x} d t+s \int_{Q_{T}}\left(\nabla \cdot\left(\varepsilon_{r} E\right)\right)(\nabla \cdot \lambda) d \mathbf{x} d t-\int_{\partial S_{T}} \lambda p d \sigma d t
\end{aligned}
$$


where $E$ and $\lambda$ are weak solutions of problems (26)-(28) and (29)-(31), respectively.

Clearly, (32) implies that the sum of integral terms in (34) equals zero. Hence, $L\left(E, \lambda, \varepsilon_{r}\right)=F\left(E, \varepsilon_{r}\right)$. In (34) $\left(E, \lambda, \varepsilon_{r}\right)=w \in U^{1}$ and functions $E$ and $\lambda$ depend on the coefficient $\varepsilon_{r}$. To get the Fréchet derivative $L^{\prime}$ of the Lagrangian (34) rigorously, one should assume that variations of functions $u$ and $\lambda$ depend on variations of the coefficient $\varepsilon_{r}$, similarly with section 4.8 of [6] and we will do that in our future publications. In this work, to derive the Fréchet derivative of the Lagrangian (34) we assume for brevity that in (34) the vector function $\left(E, \lambda, \varepsilon_{r}\right)$ can be varied independently of each other. Thus, we search a point $w \in U^{1}$ such that

$$
L^{\prime}(w)(\bar{w})=0, \quad \forall \bar{w} \in U^{1} .
$$

To find the Fréchet derivative $L^{\prime}(w)$, we consider $L(w+\bar{w})-L(w), \forall \bar{w} \in U^{1}$ and single out the linear, with respect to $\bar{w}$, part of the obtained expression. Thus, using (32), (33) and (24), we obtain

$$
L^{\prime}(w)(\mathbf{x})=\gamma\left(\varepsilon_{r}-\varepsilon_{r, g l o b}\right)(\mathbf{x})-\int_{0}^{T} \frac{\partial \lambda}{\partial t} \frac{\partial E}{\partial t}(\mathbf{x}, t) d t+s \int_{0}^{T}(\nabla \cdot E)(\nabla \cdot \lambda)(\mathbf{x}, t) d t, \mathbf{x} \in G_{b} .
$$

\section{Finite element discretization}

Consider a partition $K_{h}=\{K\}$ of $G_{b}$ which consists of tetrahedra with a mesh function $h$ defined as $\left.h\right|_{K}=h_{K}$ - the local diameter of the element $K$. Let $J_{\tau}=\{J\}$ be a partition of the time interval $(0, T)$ into subintervals $J=\left(t_{k-1}, t_{k}\right]$ of uniform length $\tau=t_{k}-t_{k-1}$. We also assume the minimal angle condition on the $K_{h}[12$.

To formulate the finite element method for solving the state problem (26)-(28) and the adjoint problem (29) -(31), and to compute the gradient of the Lagrangian via (36), we define the finite element spaces $V_{h} \subset L_{2}\left(G_{b}\right), W_{h}^{E} \subset H_{E}^{1}\left(Q_{T}\right)$ and $W_{h}^{\lambda} \subset H_{\lambda}^{1}\left(Q_{T}\right)$. First, we introduce the finite element trial space $W_{h}^{E}$ for every component of the electric field $E$ defined by

$$
W_{h}^{E}:=\left\{w \in H_{E}^{1}:\left.w\right|_{K \times J} \in P_{1}(K) \times P_{1}(J), \forall K \in K_{h}, \forall J \in J_{\tau}\right\},
$$

where $P_{1}(K)$ and $P_{1}(J)$ denote the set of linear functions on $K$ and $J$, respectively. We also introduce the finite element test space $W_{h}^{\lambda}$ defined by

$$
W_{h}^{\lambda}:=\left\{w \in H_{\lambda}^{1}:\left.w\right|_{K \times J} \in P_{1}(K) \times P_{1}(J), \forall K \in K_{h}, \forall J \in J_{\tau}\right\} .
$$

Hence, the finite element spaces $W_{h}^{E}$ and $W_{h}^{\lambda}$ consist of continuous piecewise linear functions in space and time. To approximate the function $\varepsilon_{r}(\mathbf{x})$, we use the space of piecewise constant functions $V_{h} \subset L_{2}(\Omega)$,

$$
V_{h}:=\left\{u \in L_{2}(\Omega):\left.u\right|_{K} \in P_{0}(K), \forall K \in K_{h}\right\},
$$

where $P_{0}(K)$ is the set of piecewise constant functions on $K$. In our computations we truncate computed functions $\varepsilon_{r}(\mathbf{x})$ to unity outside of the domain $\Omega_{F E M}=\Omega$ using (5) as

$$
\bar{\varepsilon}_{r}(\mathbf{x})=\left\{\begin{array}{c}
\varepsilon_{r}(\mathbf{x}), \mathbf{x} \in \Omega_{F E M}, \\
1, \mathbf{x} \in \Omega_{F D M}
\end{array}\right.
$$

Next, we set $U_{h}=W_{h}^{E} \times W_{h}^{\lambda} \times V_{h}$. Obviously $\operatorname{dim} U_{h}<\infty$ and $U_{h} \subset U^{1}$ as a set. Because of this, we consider $U_{h}$ as a discrete analogue of the space $U^{1}$. We introduce the same norm in $U_{h}$ as the one in $U^{0},\|\cdot\|_{U_{h}}:=\|\cdot\|_{U^{0}}$, with

$$
U^{0}=L_{2}\left(G_{T}\right) \times L_{2}\left(G_{T}\right) \times L_{2}(\Omega) .
$$

The finite element method for solving equation (35) now reads: Find $u_{h} \in U_{h}$, such that

$$
L^{\prime}\left(u_{h}\right)(\bar{u})=0, \forall \bar{u} \in U_{h} .
$$




\section{Mesh refinement recommendation and the adaptive algorithm}

From Theorem 5.1 and Remark 5.1 of [10] it follows that the finite element mesh should be locally refined in such subdomain of $\Omega$ where the maximum norm of the Frëchet derivative of the objective functional is large. For each mesh we first linearly interpolate the coefficient $\varepsilon_{r, g l o b}(\mathbf{x})$ on it, and use the interpolated coefficient as an initial guess for on the current mesh. Our algorithm consists of two loops: the outer loop deals with the locally adaptive mesh refinement. In the inner loop, i.e., on each mesh, we iteratively update the approximations $\varepsilon_{h}^{m}$ of the function $\varepsilon_{h}$ by solving (35) using an optimization procedure, where $m$ is the index of iteration in the optimization procedure. Denote by

$$
L_{h}^{\prime, m}(\mathbf{x})=-\int_{0}^{T} \frac{\partial \lambda_{h}^{m}}{\partial t} \frac{\partial E_{h}^{m}}{\partial t} d t+s \int_{0}^{T} \nabla \cdot E_{h}^{m} \nabla \cdot \lambda_{h}^{m} d t+\gamma\left({\overline{\varepsilon_{h}}}^{m}-\bar{\varepsilon}_{r, g l o b}\right) .
$$

\section{Adaptive algorithm}

Step 0. Choose an initial mesh $K_{h}$ in $\Omega$ and an initial time partition $J_{0}$ of the time interval $(0, T)$. Start from the initial guess $\varepsilon_{h}^{0}=\varepsilon_{r, g l o b}$, we compute the approximations $\varepsilon_{h}^{m}$ via the following steps:

Step 1. Compute the solutions $E_{h}\left(x, t, \varepsilon_{h}^{m}\right)$ and $\lambda_{h}\left(x, t, \varepsilon_{h}^{m}\right)$ of the state problem (15)-(18) and the adjoint problem (29) - (31) on $K_{h}$ and $J_{k}$, and compute the Frëchet derivative $L_{h}^{\prime, m}$ via (40).

Step 2. Update the coefficient on $K_{h}$ and $J_{k}$ using the conjugate gradient method:

$$
\varepsilon_{h}^{m+1}:=\varepsilon_{h}^{m}+\alpha d^{m}(\mathbf{x}),
$$

where $\alpha>0$ is a step-size in the conjugate gradient method, given by a line search procedure, see, e.g., [24], and

$$
d^{m}(\mathbf{x})=-L_{h}^{\prime, m}(\mathbf{x})+\beta^{m} d^{m-1}(\mathbf{x})
$$

with

$$
\beta^{m}=\frac{\left\|L_{h}^{\prime, m}\right\|^{2}}{\left\|L_{h}^{\prime, m-1}\right\|^{2}}
$$

where $d^{0}(\mathbf{x})=-L_{h}^{\prime, 0}(\mathbf{x})$.

Step 3. Stop updating the coefficient and set $\varepsilon_{h}:=\varepsilon_{h}^{m+1}, M:=m+1$, if either $\left\|L_{h}^{\prime, m}\right\|_{L_{2}(\Omega)} \leq \theta$ or norms $\left\|\varepsilon_{h}^{m}\right\|_{L_{2}(\Omega)}$ are stabilized. Here $\theta$ is a tolerance number. Otherwise, set $m:=m+1$ and go to step 1 .

Step 4. Compute $L_{h}^{\prime, M}$ via (40). Refine the mesh at all grid points $\mathbf{x}$ where

$$
\left|L_{h}^{\prime, M}(\mathbf{x})\right| \geq \beta_{1} \max _{\bar{\Omega}}\left|L_{h}^{\prime, M}(\mathbf{x})\right| .
$$

Here the tolerance number $\beta_{1} \in(0,1)$ is chosen by the user.

Step 5. Construct a new mesh $K_{h}$ in $\Omega$ and a new time partition $J_{k}$ of the time interval $(0, T)$. On $J_{k}$ the new time step $\tau$ should be chosen in such a way that the CFL condition is satisfied. Interpolate the initial approximation $\varepsilon_{r, g l o b}$ from the previous mesh to the new mesh. Next, return to step 1 at $m=1$ and perform all above steps on the new mesh.

Step 6. Let $\left\|L_{h, \text { prev }}^{\prime, M}\right\|_{L_{2}(\Omega)}$ and $\left\|L_{h, \text { current }}^{\prime, M}\right\|_{L_{2}(\Omega)}$ be norms defined in step 4 on the previous and current mesh, respectively. Stop mesh refinements if $\left\|L_{h, \text { current }}^{\prime, M}\right\|_{L_{2}(\Omega)} \geq\left\|L_{h, \text { prev }}^{\prime, M}\right\|_{L_{2}(\Omega)}$. 


\section{Some details of numerical implementation}

In this section we present results of reconstruction of dielectric constants/refractive indices and shapes of some targets using the adaptivity algorithm of section [6. One of the discrepancies between our mathematical model (15) - (20) and the measured experimental data is that formally equation (15) is invalid for the case when metallic targets are present (we refer to [8] for the description of other discrepancies). However, it was shown computationally in [16] that one can treat metallic targets as dielectrics with large dielectric constants.

We call these effective (or "appearing") dielectric constants and values for them are in the interval

$$
\varepsilon_{r}(\text { metallic target }) \in(10,30) \text {. }
$$

Modeling metallic targets as integral parts of the unknown coefficient $\varepsilon_{r}(\mathbf{x})$ is convenient for our practical computations in order to image IEDs. Since IEDs usually consist of mixtures of some dielectrics with a number of metallic parts, these targets are heterogeneous ones, and we consider three heterogeneous cases in section 8. However, modeling metallic parts separately from dielectric ones is impractical for our application because of those mixtures.

Using (42), we define in all our tests the upper value of the function $\varepsilon_{r}(\mathbf{x})$ as $d=25$, see (3). Thus, we set lower and upper bounds for the reconstructed function $\varepsilon_{r}(\mathbf{x})$ in $\Omega$ as

$$
M_{\varepsilon_{r}}=\left\{\varepsilon_{r}(\mathbf{x}): \varepsilon_{r}(\mathbf{x}) \in[1,25]\right\} .
$$

In our computation we ensure lower and upper bounds via truncating those values of $\varepsilon_{r}(\mathbf{x})$ which are outside of the interval (43).

\subsection{Dirichlet and Neumann boundary conditions}

In our experiments only one component $E_{2}(\mathbf{x}, t)$ of the electric field $E(\mathbf{x}, t)=\left(E_{1}, E_{2}, E_{3}\right)(\mathbf{x}, t)$ is both sent into the medium and measured. Thus, only the second component of the function $g$ in the Dirichlet boundary condition (41) on $\Gamma$ is available. We approximate the other two components of $g$ on $\Gamma$ by the numerical solution of the forward problem (15)-(20), with the coefficient given by $\varepsilon_{r}=\varepsilon_{r, g l o b}(\mathbf{x})$ - the solution of the globally convergent method. The Dirichlet data on the rest of the boundary $\partial G_{b}$, i.e., on $\partial G_{b} \backslash \Gamma$, as well as the Neumann condition $p(\mathbf{x}, t)$ in (25) at the entire boundary $\partial G_{b}$ are taken from the numerical solution of that forward problem.

\subsection{Computational domains}

To generate the boundary data (23), (25) for all three components of the electric field $E$, as specified in the previous section, we solve the forward problem in the computational domain $G$, which we choose as

$$
G=\{\mathbf{x}=(x, y, z) \in(-0.56,0.56) \times(-0.56,0.56) \times(-0.16,0.1)\} .
$$

The boundary of the domain $G$ is $\partial G=\partial_{1} G \cup \partial_{2} G \cup \partial_{3} G$. Here, $\partial_{1} G$ and $\partial_{2} G$ are front and back sides of the domain $G$ at $\{z=0.1\}$ and $\{z=-0.16\}$, respectively, and $\partial_{3} G$ is the union of left, right, top and bottom sides of this domain.

We use a stabilized domain decomposition method of [9] implemented in the software package WavES 30. The FEM domain $\Omega_{F E M}$ is chosen as

$$
\Omega_{F E M}=\Omega=\{\mathbf{x}=(x, y, z) \in(-0.5,0.5) \times(-0.5,0.5) \times(-0.1,0.04)\} .
$$

After the data propagation procedure, see discussions in section 7.3 , the data $g(\mathbf{x}, t)$ in (4) are given at the front side $\Gamma$ of the domain $\Omega$ which is defined as

$$
\Gamma=\{\mathbf{x} \in \partial \Omega: z=0.04\} .
$$


The waveform function $f(t)$ in our simulated incident plane wave is chosen as

$$
f(t)=\sin \omega t, 0 \leq t \leq t_{1}:=\frac{2 \pi}{\omega} .
$$

Here, we initialize the plane wave at $\{z=0.1\}$. We use $\omega=30, T=1.2$ and $s=1$. We solve the problem (15) - (20) using the explicit scheme of [9] with the time step size $\tau=0.003$, which satisfies the CFL condition. Note that this time step is dimensionless, which corresponds to the time step of $10 \mathrm{ps}$ in our real experiment. Here we use the dimensionless time step in order to normalize the coefficient $\varepsilon_{r}$ to be unity outside of $\Omega$. The dimensionless time $T=1.2$ corresponds to $4 \mathrm{~ns}$ in our real time. We do not use the whole $10 \mathrm{~ns}$ recorded data since after data preprocessing, they are shifted earlier in time and they do not contain any target's signal after 4 ns.

\subsection{Data Preprocessing}

In the previous section, we implicitly assumed that our model is comparable to the experimental data. Moreover, the data is available at the plane $\Gamma$ of $\Omega$, which is quite close to the targets. Unfortunately, this is not the case in practice. In fact, there is a huge misfit bewteen our experimental data and the simulated ones, see [26. Therefore, data preprocessing is required in order to prepares the experimental data to become an input for our inversion algorithm. In our experience, data preprocessing of experimental data is always a heuristic procedure. That procedure for the globally convergent method was described in detail in [26. Since the globally convergent method works with the PDE which is obtained by the Laplace transform of the original wave-like equation (11), and we work here directly in time domain, we need less number of data preprocessing steps than in [26. We only describe the following preprocessing steps which are different from those of [26].

\subsubsection{Data propagation}

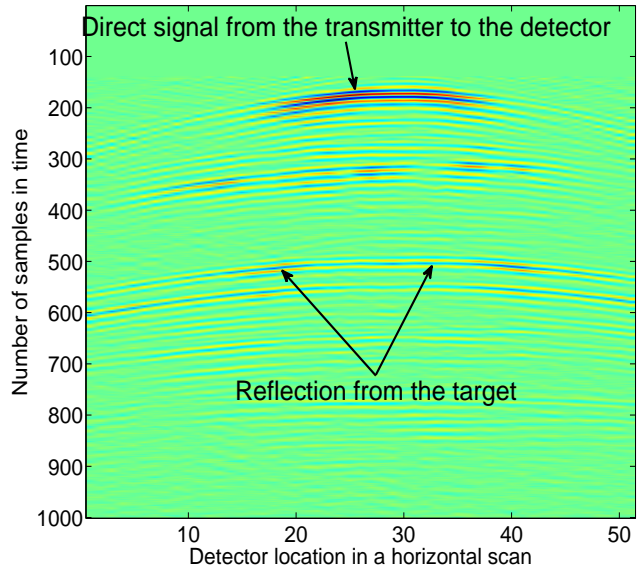

(a)

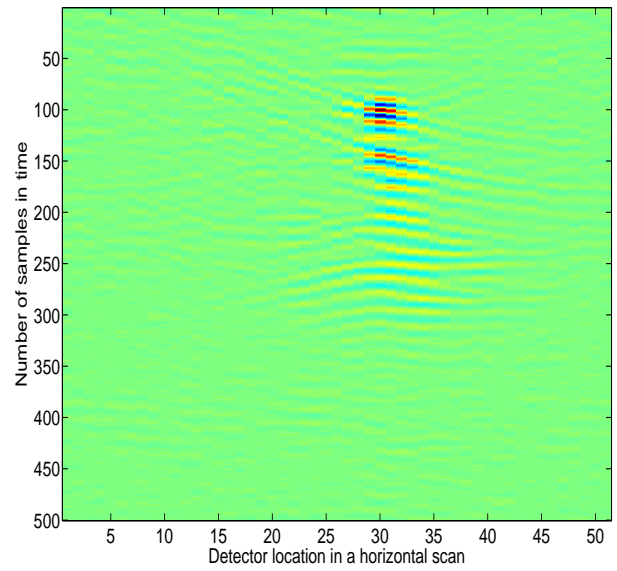

(b)

Figure 2: Result of data propagation for target number 1 (Table 1). Horizontal axis is the indices of the detector's locations and vertical axis is number of samples in time. a) data at the measurement plane, b) data at the propagated plane. One can see that the propagated data are focused at the target, whereas original data are smeared out.

In [26, we used a time-reversal data propagation method in order to migrate our data from the measurement plane, which is in the far field zone, to the plane $\Gamma$, which is at about $4 \mathrm{~cm}$ far from the 
targets. In this paper, we use another data propagation method based on the Fourier transform. This technique is basically the same as the Stolt migration in Geophysics, see [25, 31. However, in the standard Stolt migration the wave at the initial time is calculated in the whole spatial domain of interest, whereas we calculate the wave only at a plane parallel to the measurement plane but in the whole time interval. The technique is described in detail in [27.

A result of the data propagation is illustrated in Figure 2. The figure shows a horizontal scan of target number 1, see Table 1. The horizontal axis is the indices of the detector's locations and the vertical axis is the number of samples in time. Time increases from the top to the bottom. Figure 2 (a) shows the original data while Figure 2(b) shows the data after the propagation. As can be seen from these figures, the target's signas in the original data is smeared out. On the other hand, it is focused after the data propagation.

\subsubsection{Data calibration}

Since the amplitude of experimental data are very different from that of computational simulations, we must multiply the experimental data by a calibration factor $\theta$ so that they have similar amplitude as the simulated data. This factor is not easy to obtain in practice and we should somehow find an appropriate one. The calibration of the measured data was done in the Laplace transform domain in [26]. However, here we work with the time domain data only. Therefore, we use a new data calibation procedure we described below.

Let the function $g_{\text {exper }}(\mathbf{x}, t), \mathbf{x} \in \Gamma, t \in(0, T)$ be our propagated experimental data. This function is given only on grid points $\left(\mathbf{x}_{i}, t_{j}\right)$. We compute the maximal value of this function,

$$
g_{\max }=\max _{\left(\mathbf{x}_{i}, t_{j}\right)} g_{\text {exper }}\left(\mathbf{x}_{i}, t_{j}\right)
$$

Usually the number $g_{\max }$ is quite large. Next, let $E_{2, \operatorname{sim}}(\mathbf{x}, t)$ be the function which is computed via solving the problem (15)-(20). We compute the maximal value of this function on $\Gamma$,

$$
E_{2, \max }=\max _{\Gamma \times[0, T]} E_{2, \operatorname{sim}}(\mathbf{x}, t)
$$

Define $r=E_{2, \max } / g_{\max }$. Next, we assign

$$
g_{\text {incl }}\left(\mathbf{x}_{i}, t_{j}\right):=r \cdot g_{\text {exper }}\left(\mathbf{x}_{i}, t_{j}\right)
$$

and use the function $g_{\text {incl }}\left(\mathbf{x}_{i}, t_{j}\right)$ as the second component of the vector function $g(\mathbf{x}, t)$ in (4). Two other steps of data preprocessing are due to (23) and (25) in section 4. The final step of data preprocessing, the so-called "immersing procedure" is done as follows.

\subsubsection{Data immersing}

In this section we describe a heuristic immersing procedure of the time-dependent propagated experimental data $g_{\text {incl }}(\mathbf{x}, t)=\left.E_{2}(\mathbf{x}, t)\right|_{\mathbf{x} \in \Gamma}$. This procedure does two things:

- immerses the data $g_{\text {incl }}(\mathbf{x}, t)$ into computationally simulated ones;

- extends the data $g_{i n c l}(\mathbf{x}, t)$ from $\Gamma$ to $\Gamma_{1}$.

By (45) the rectangle $\Gamma$ is smaller than the rectangle

$$
\Gamma_{1}=\{\mathbf{x}:(x, y) \in(-0.56,0.56) \times(-0.56,0.56), z=0.04\} .
$$

It is clear from the adjoint problem (29)-31) that we need to get a proper data for the function $E_{2}(\mathbf{x}, t)$ for $(\mathbf{x}, t) \in \Gamma_{1} \times(0, T)$ while having the data $E_{2}(\mathbf{x}, t)=g_{\text {incl }}(\mathbf{x}, t)$ only for $\mathbf{x} \in \Gamma$. We now describe how do we extend the data from $\Gamma$ to $\Gamma_{1}$. Let $E_{2}(\mathbf{x}, t)$ be the $E_{2}$-component of the solution $E(\mathbf{x}, t)$ of 
the forward problem (15) 201 with $\varepsilon_{r}:=\varepsilon_{r, g l o b}$. Then we define our immersed function $E_{2}^{\text {immers }}(\mathbf{x}, t)$ for $(\mathbf{x}, t) \in \Gamma_{1} \times(0, T)$ as

$$
E_{2}^{\text {immers }}(\mathbf{x}, t)= \begin{cases}g_{\text {incl }}(\mathbf{x}, t), & \text { if } \mathbf{x} \in \Gamma \text { and } g_{\text {incl }}(\mathbf{x}, t) \geq \beta \max _{\bar{\Gamma}} g_{\text {incl }}(\mathbf{x}, t), \\ E_{2}(\mathbf{x}, t), & \text { if } \mathbf{x} \in \Gamma \text { and } g_{\text {incl }}(x, t)<\beta \max _{\bar{\Gamma}} g_{\text {incl }}(\mathbf{x}, t), \\ E_{2}(\mathbf{x}, t), & \text { if } \mathbf{x} \in \Gamma_{1} \backslash \Gamma .\end{cases}
$$

We choose the parameter $\beta \in(0,1)$ in (47) in numerical experiments of section 8 . It follows from (47) that $E_{2}^{\text {immers }}(\mathbf{x}, t)=E_{2}(\mathbf{x}, t)$ for $\mathbf{x} \in \Gamma_{1} \backslash \Gamma$.

Figures 3. 4 show that, depending on the parameter $\beta$ in (47), the data immersing procedure not only allows to extend the data from $\Gamma$ to $\Gamma_{1} \backslash \Gamma$ but also make the experimental data usable in our inverse algorithm. Indeed, we note that the experimental data is measured at a very high frequency, say, $\omega \approx 170$, whereas our simulations are done at $\omega=30$ in order to reduce the computational cost. Therefore, the experimental data are not compatible with the simulations. Our immersing procedure helps to avoid solving the problem at a very high frequency. After this immersing procedure we solve the inverse problem using the algorithm of section 6 .

\subsection{Postprocessing of results}

Results of the globally convergent algorithm of the first stage procedure have demonstrated that this algorithm provides accurate locations of targets as well as accurate values of refractive indices $n=\sqrt{\varepsilon_{r}}$ of dielectric targets and large values of appearing dielectric constants $\varepsilon_{r}$ for metallic targets [8, 26]. However, it does not reconstruct shapes of targets well, especially the size in the $z$-direction. The latter is the reason why we apply the second stage to refine results of the first.

Let $\varepsilon_{r}(\mathbf{x})$ be the function obtained in the adaptive algorithm of section 6 . We form the image of the dielectric targets based on the function $\varepsilon_{r, \text { diel }}(\mathbf{x})$,

$$
\varepsilon_{r, \text { diel }}(\mathbf{x})=\left\{\begin{array}{l}
\varepsilon_{r}(\mathbf{x}) \text { if } \varepsilon_{r}(\mathbf{x}) \geq 0.85 \max _{\bar{\Omega}} \varepsilon_{r}(\mathbf{x}) \\
1 \text { otherwise }
\end{array}\right.
$$

As to the metallic targets (i.e., the ones with large computed maximal values of $\varepsilon_{r}(\mathbf{x})$ ), we use the function $\varepsilon_{r, \text { metal }}(\mathbf{x})$,

$$
\varepsilon_{r, \text { metal }}(\mathbf{x})=\left\{\begin{array}{l}
\varepsilon_{r}(\mathbf{x}) \text { if } \varepsilon_{r}(\mathbf{x}) \geq 0.3 \max _{\bar{\Omega}} \varepsilon_{r}(\mathbf{x}) \\
1 \text { otherwise }
\end{array}\right.
$$

\section{Reconstruction results}

In our numerical studies we apply adaptive algorithm of section 6 to refine shape for nine (9) targets listed in Table 1. Three of them (targets number 1,2,5) were dielectrics, three were metallic objects (targets number $4,5,6$ ) and three (targets number $7,8,9$ ) were dolls with different objects placed inside them. Heterogeneous targets present models for explosive devices in which explosive materials are masked by dielectrics. Target number 7 was a wooden doll which was empty inside, target number 8 was a piece of a metal inserted inside that doll, and in target number 9 dry sand was partly inserted inside the doll.

In all tests we have used the regularization parameter $\gamma=0.01$. The target number 1 was used for the calibration to choose optimal parameters for dielectrics. We choose the cut-off number in (48) to be 0.85 and the number $\beta=0.5$ in (47) for all dielectrics of Table 1 . The target number 4 was used for calibration purpose of metallic targets: we choose the cut-off number 0.3 in (49). In the case of metals we have used the same numbers $\beta=0.5$ in (47) as for dielectrics.

Figures 3. 4. show backscattered immersed data of the second component $E_{2}$ of the electric field for object 7 at different times and with different immersing factor $\beta$ in (47).

In the case of dielectric targets we have a posteriori directly measured their refractive indices $n=\sqrt{\varepsilon_{r}}$. Let $\varepsilon_{r}^{c o m p}(\mathbf{x})$ be the computed coefficient. We consider maximal values of functions $\varepsilon_{r}^{c o m p}(\mathbf{x})$. This means 


\begin{tabular}{|c|l|}
\hline Target number & Specification of the target \\
\hline 1 & a piece of oak, rectangular prism \\
2 & a piece of pine \\
3 & a metallic sphere \\
4 & a metallic cylinder \\
5 & a piece of oak \\
6 & a metallic rectangular prism \\
7 & a wooden doll, air inside, heterogeneous target \\
8 & a wooden doll, metal inside, heterogeneous target \\
9 & a wooden doll, sand inside, heterogeneous target \\
\hline
\end{tabular}

Table 1: Names of targets.

\begin{tabular}{|l|l|l|l|l|l|c|}
\hline Target number & 1 & 2 & 5 & 7 & 9 & Average error \\
\hline Measured $n$, error & $2.11,19 \%$ & $1.84,18 \%$ & $2.14,28 \%$ & $1.89,30 \%$ & $2.1,26 \%$ & $24 \%$ \\
\hline$n$ in glob.conv, error & $1.92,9 \%$ & $1.8,2 \%$ & $1.83,15 \%$ & $1.86,2 \%$ & $1.92,9 \%$ & $8 \%$ \\
\hline$n$, coarse mesh, error & $1.94,8 \%$ & $1.82,1 \%$ & $1.84,14 \%$ & $1.88,0.5 \%$ & $1.93,8 \%$ & $6 \%$ \\
\hline$n, 1$ time ref. mesh, error & $1.94,8 \%$ & $1.82,1 \%$ & $1.85,14 \%$ & $1.89,0 \%$ & $1.93,8 \%$ & $6 \%$ \\
\hline$n, 2$ times ref.mesh, error & & $1.84,0 \%$ & & $1.9,0.5 \%$ & $1.96,7 \%$ & $2 \%$ \\
\hline$n, 3$ times ref.mesh, error & & & & $1.89,0 \%$ & & $0 \%$ \\
\hline
\end{tabular}

Table 2: Computed n(target) and directly measured refractive indices of dielectric targets together with both measurement and computational errors as well as the average error. Note that the average computational errors are at least 4 times less than the average error of direct measurements. In all tests we have used the following values of above parameters: the regularization parameter $\gamma=0.01$ in (24), $\theta=10^{-9}$ in Step 3 of the adaptive algorithm, $\beta_{1}=0.7$ in (41) and $s=1$ in (15).

\begin{tabular}{|l|l|l|l|l|}
\hline Target number & 3 & 4 & 6 & 8 \\
\hline$\varepsilon_{r}$ (target $)$ of glob.conv. & 14.4 & 15.0 & 25 & 13.6 \\
\hline$\varepsilon_{r}$ (target $)$ coarse mesh & 14.4 & 17.0 & 25 & 13.6 \\
\hline$\varepsilon_{r}($ target $) 1$ time ref.mesh & 14.5 & 17.0 & 25 & 13.6 \\
\hline$\varepsilon_{r}$ (target $) 2$ times ref.mesh & 14.6 & 17.0 & 25 & 13.7 \\
\hline$\varepsilon_{r}$ (target $) 3$ times ref.mesh & 14.6 & 17.0 & & 14.0 \\
\hline$\varepsilon_{r}($ target $) 4$ times ref.mesh & & 17.0 & & \\
\hline
\end{tabular}

Table 3: Computed appearing dielectric constants $\varepsilon_{r}$ (target) of metallic targets with numbers 3,4,6 as well as of target number 8 which is a metal covered by a dielectric. The mesh refinement process for target number 6 has stopped on three (3) mesh refinements. 


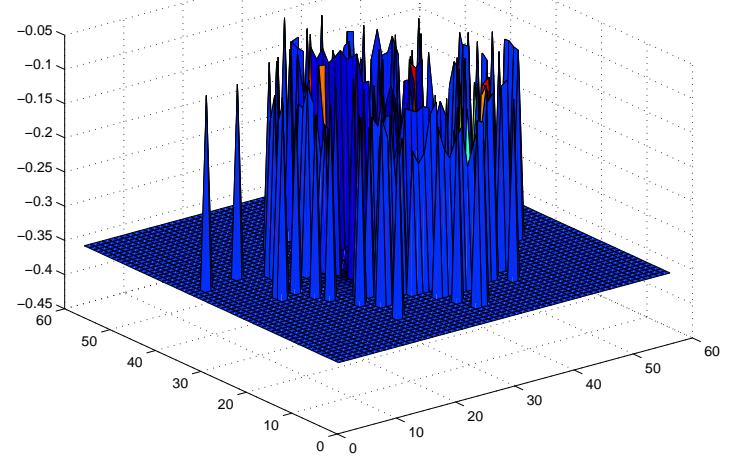

(a) $\beta=0.2, t=0.3$

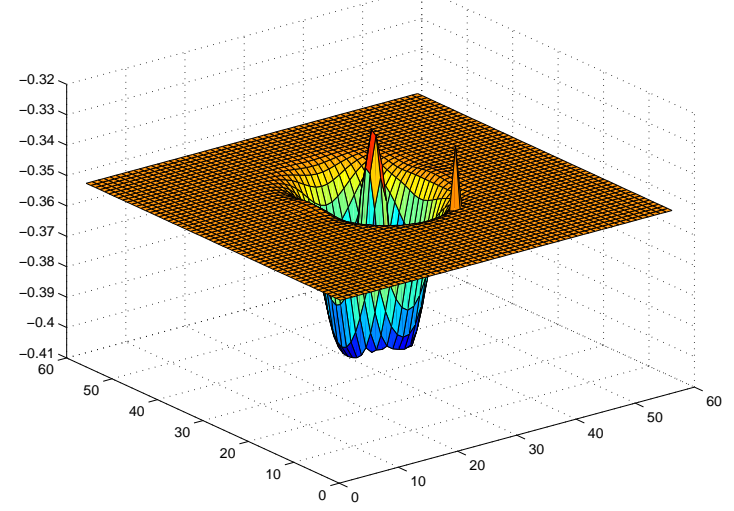

(c) $\beta=0.8, t=0.3$

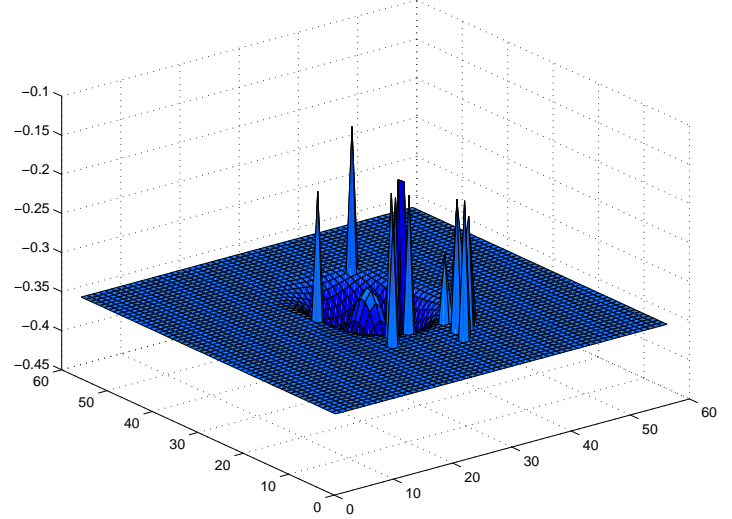

(b) $\beta=0.5, t=0.3$

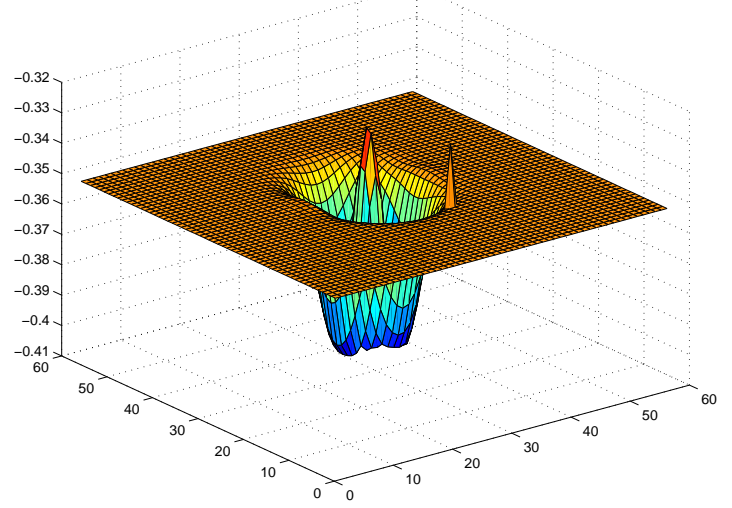

(d) $\beta=0.9, t=0.3$

Figure 3: Backscattered immersed data of the second component $E_{2}$ of electric field for object number 7 (wooden doll, empty inside) of Table 1 for different values of the parameter $\beta$ in (47). Recall that the final time is $T=1.2$.

that in our Tables 2, 3 we list values of dielectric constants $\varepsilon_{r}$ (target) and refractive indices $n$ (target) as

$$
\left.\varepsilon_{r}(\text { target })=\max _{\bar{\Omega}} \varepsilon_{r}^{c o m p}(\mathbf{x}), n \text { (target }\right)=\sqrt{\varepsilon_{r}(\text { target })} .
$$

Tables 2, 3 are quite informative ones since they show the accuracy of our reconstruction of either refractive indices (Table 2) or effective dielectric constants (Table 3). Table 2 lists refractive indices of dielectric targets, both computed $n$ (target) and directly measured ones $n$. Computed numbers $n$ (target) are displayed for different locally refined meshes. This table also shows the measurement error in direct measurements of $n$. Table 3 lists calculated effective dielectric constants $\varepsilon_{r}$ (target) of metallic targets, again for different locally refined meshes. In Table 3 for all metallic targets we have $\varepsilon$ (target) $>10$, which means that (42) is satisfied.

One can derive several important observations from Table 2, First, for all targets and on all adaptively refined meshes the computational error is significantly less than the error of direct measurements. Thus, the average computational error is significantly less than the average measurement error on all adaptively refined meshes. Second, computed refractive indices are within reasonable error estimates in all cases. The accuracy on all adaptively refined meshes is about the same.

We observe from Table 3 that for target number 8 we have obtained effective dielectric constant $\varepsilon$ (target) $\in[13.6,14]$ on all adaptively refined meshes, which is less than for other metallic objects. We 


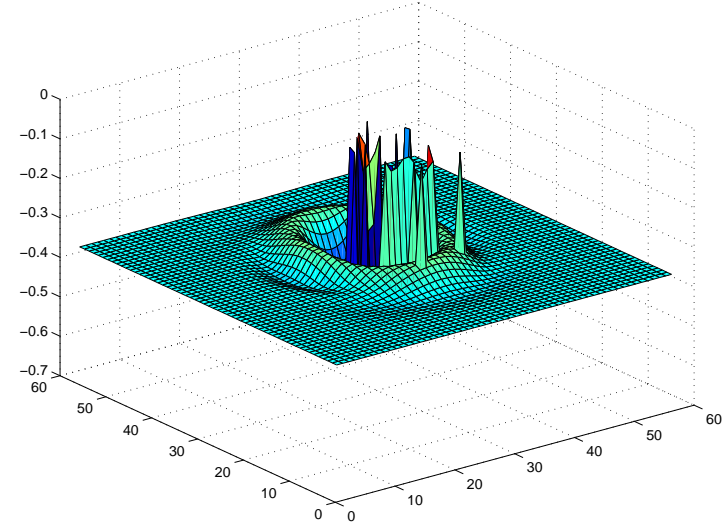

(a) $\beta=0.2, t=0.45$

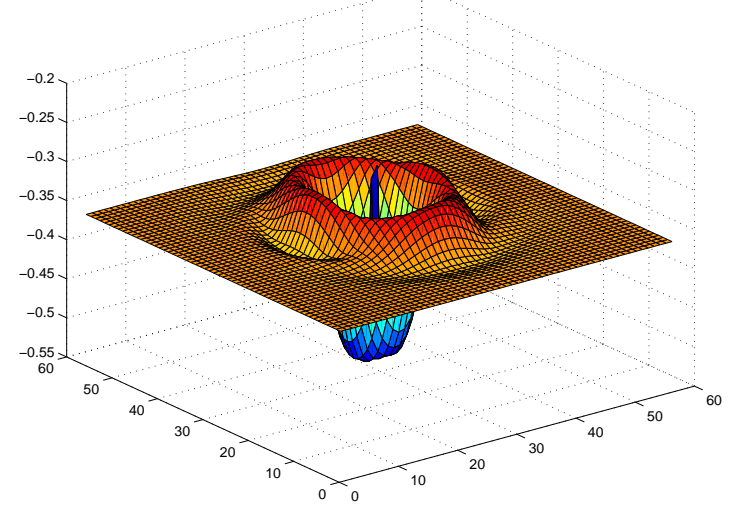

(c) $\beta=0.8, t=0.45$

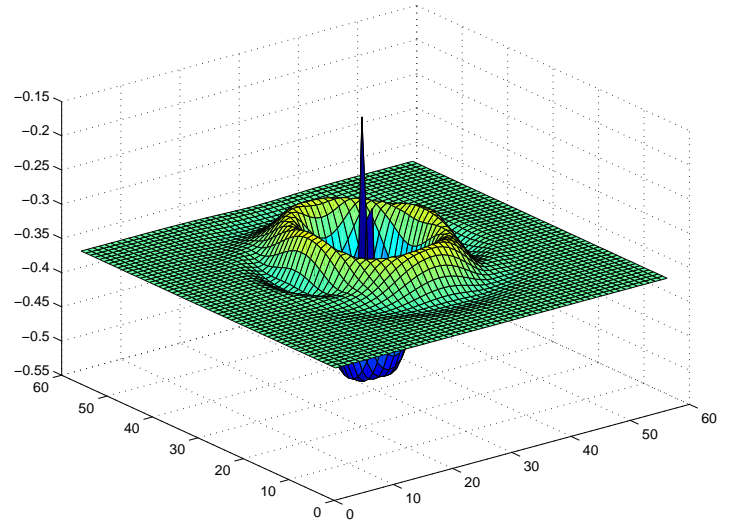

(b) $\beta=0.5, t=0.45$

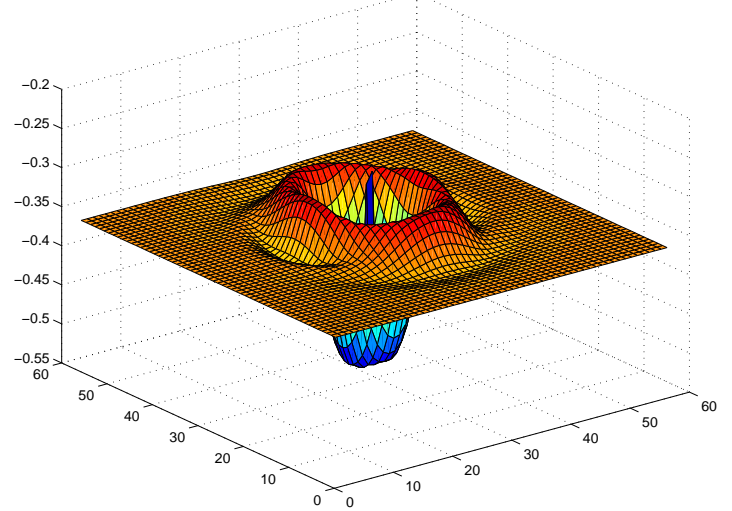

(d) $\beta=0.9, t=0.45$

Figure 4: Backscattered immersed data of the second component $E_{2}$ of electric field for object 7 (wooden doll, empty inside) of Table 1 for different values of the parameter $\beta$ in (47). Recall that the final time is $T=1.2$.

can explain this by the fact that target number 8 is a mixture of metal and dielectric. An important observation, which can be deduced from Table 3, is that our two-stage algorithm can still compute large inclusion/background contrasts exceeding 10:1, just as the algorithm of the first stage.

Figures 6 16 display 3-d images of some targets listed in Table 1 as well as corresponding adaptively locally refined meshes. To have a better visualization, we have zoomed some figures to $0.4 \times 0.4$ square from $1 \times 1$ square in the $x, y$ directions. So, in these Figures we display only the image in the domain $\Omega_{z o o m}$

$$
\Omega_{\text {zoom }}=\{\mathbf{x}=(x, y, z) \in(-0.2,0.2) \times(-0.2,0.2) \times(-0.1,0.04)\},
$$

Figures 6- 16 also show estimates of sizes of the targets in the $z$-direction. Locations of all targets as well as their sizes in $x, y, z$ directions are well estimated.

\section{Summary}

We have used time dependent backscattering experimental data generated by a single source of electric pulses to simultaneously reconstruct all three parameters of interest of explosive-like targets: their refractive indices, shapes and locations. To do this, we have used the two-stage reconstruction procedure, which was first proposed in [5. On the first stage the globally convergent method of [4, 6] was used. 


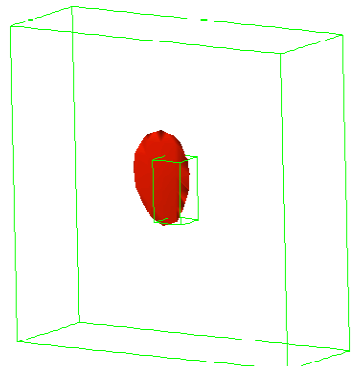

a) target 1

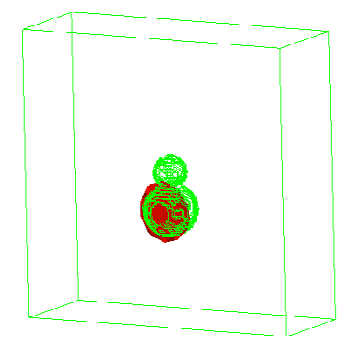

d) target 7

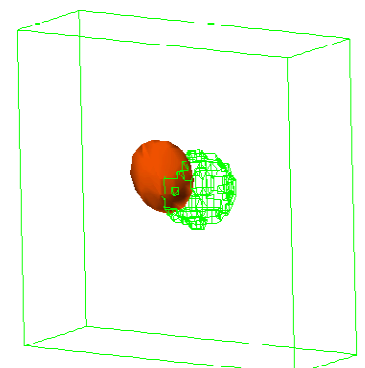

b) target 3

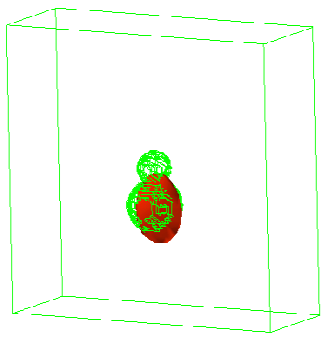

e) target 8

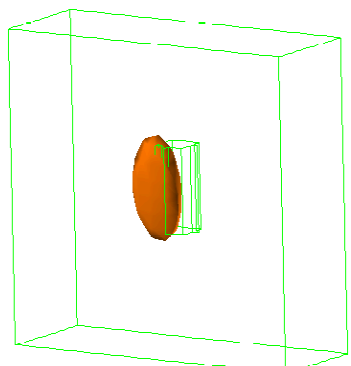

c) target 4

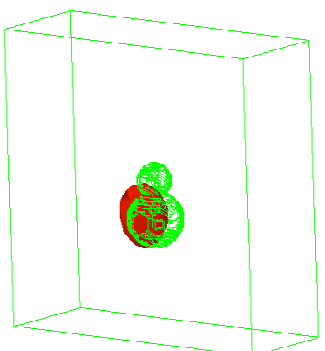

f) target 9

Figure 5: Reconstructions of some targets of Table 1 obtained in [8, 26 on the first stage of our two-stage numerical procedure.

This method has provided accurate estimates of refractive indices and locations of targets [8, 26]. On the second stage, which is the focus of this paper, images were refined using the adaptivity technique of [10. The second stage has provided accurate estimates of the third component: the shape, in addition to the first two ones. Interestingly, even heterogeneous targets, which model heterogeneous IEDs, were quite accurately imaged.

In all cases one can observe a significant improvement of the image quality after the application of the adaptivity on the second stage. Another observation here is that we can accurately image shapes of not only targets with "straight" boundaries, like a rectangular prism (target number 1) or a cylinder (target number 4) but targets with curved boundaries as well (targets number 3 and 7). Even shadow parts of targets number $1,3,4,8$ are imaged rather well. This is regardless on the fact that we use the minimal possible information content and on a narrow view angle: single location of the source and time resolved backscattering data. 


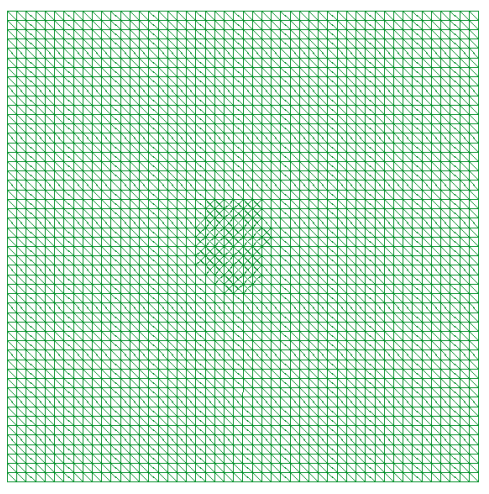

(a)

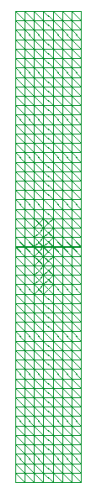

(b)

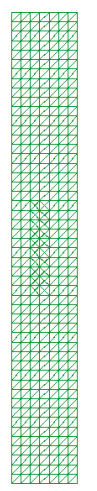

(c)

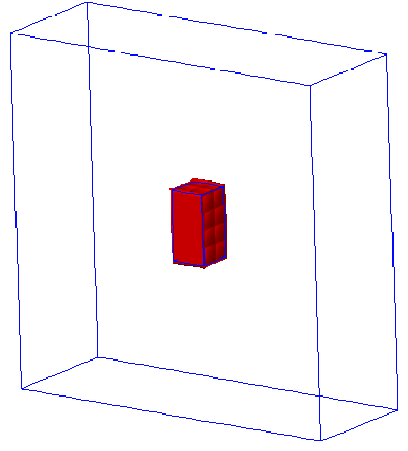

(d)

Figure 6: (a) $x y$-projection, (b) $x z$-projection, and (c) $y z$-projection of the once refined (optimal) mesh; d) Computed image of target number 1 of Table 1 on that mesh. Thin lines indicate correct shapes. To have a better visualization we have zoomed the domain $\Omega$ in (44) in the domain $\Omega_{z o o m}$ in (50). This target number 1 was used for the calibration purpose for the case of dielectric targets. A significant improvement of the image of d), compared with the image of Figure 5 -a), obtained on the first stage is evident.

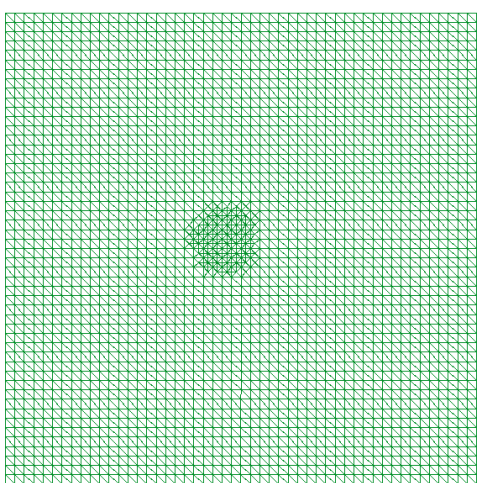

(a)

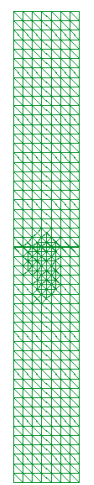

(b)

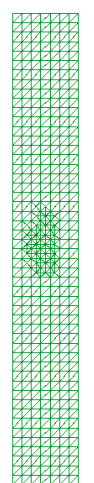

(c)

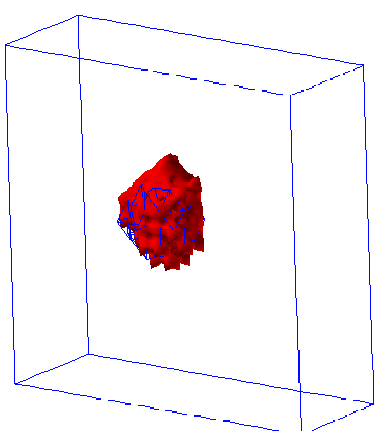

(d)

Figure 7: (a) $x y$-projection, (b) $x z$-projection, and (c) $y z$-projection of the three times refined (optimal) mesh; d) Computed image of target number 3 of Table 1 on that mesh. Thin lines indicate correct shape. To have better a visualization we have zoomed the domain $\Omega$ in (44) in the domain $\Omega_{z o o m}$ in (50). Comparison with Figure 5 b) shows a significant improvement compared with the first stage. 


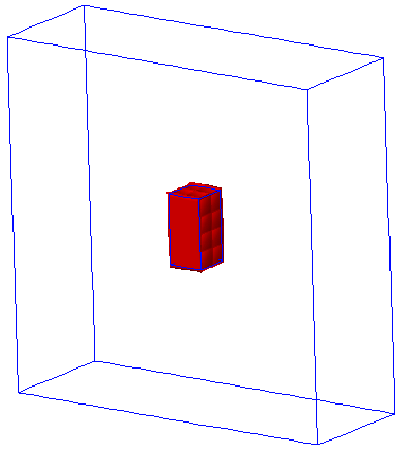

(a) Perspective view

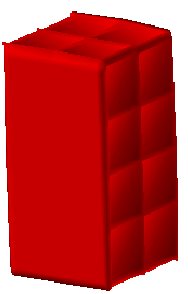

(d) Zoom, perspective

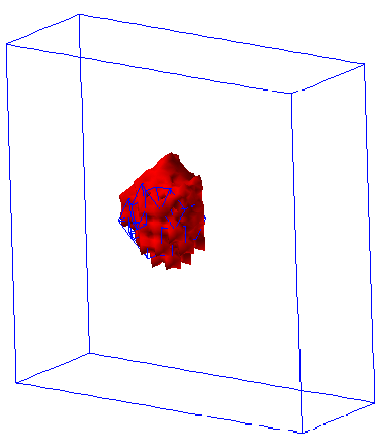

(g) Perspective view

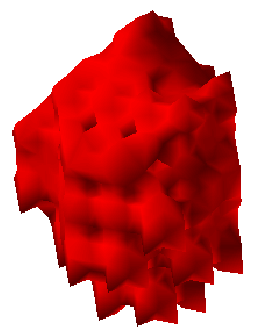

(j) Zoom, perspective

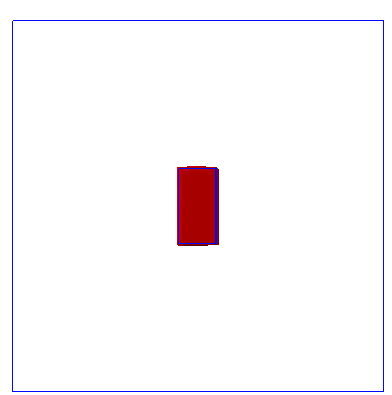

(b) Front view

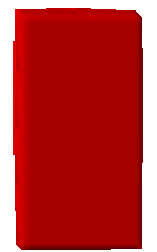

(e) Zoom, front

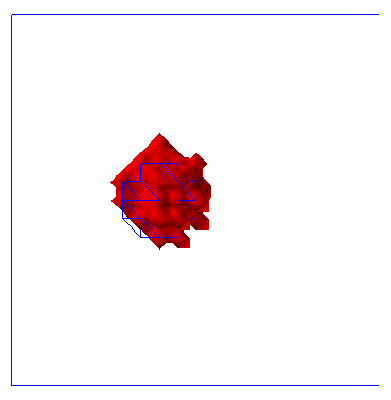

(h) Front view

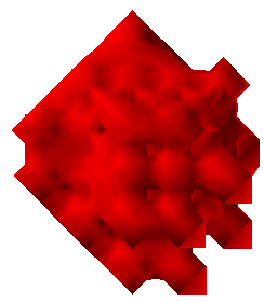

(k) Zoom, front

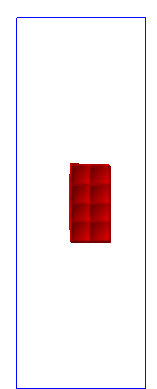

(c) Side view

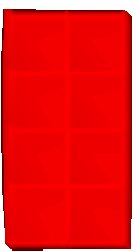

(f) Zoom, side

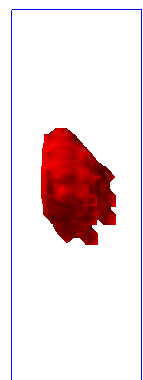

(i) Side view

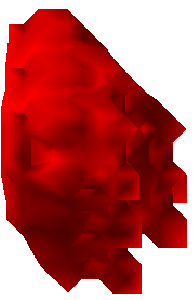

(l) Zoom, side

Figure 8: Three views and zooms of the reconstruction of target number 1 (figures a)-f)) on the once refined mesh. Three views and zooms of the reconstruction of target number 3 (figures g)-l)) of Table 1 on three times refined mesh. Recall that target number 3 is a metallic sphere. 


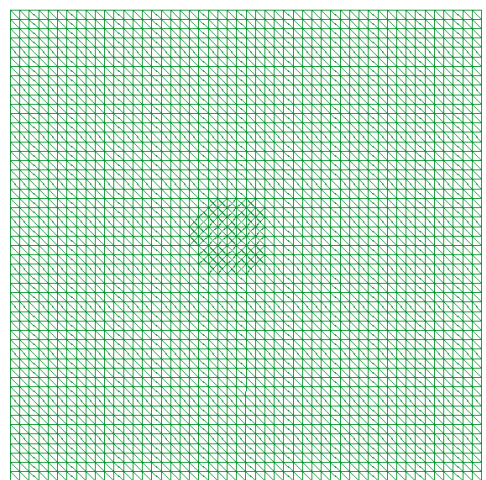

(a) $x y$-projection

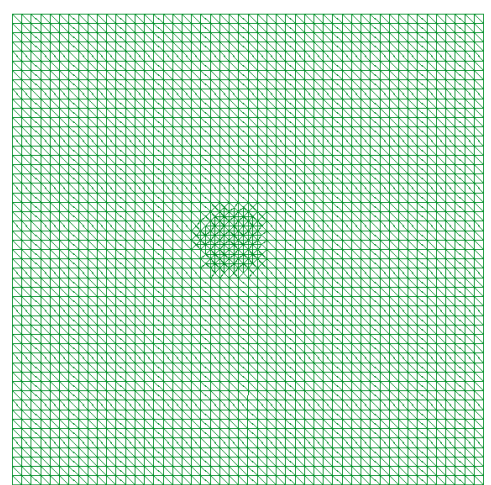

(d) $x y$-projection

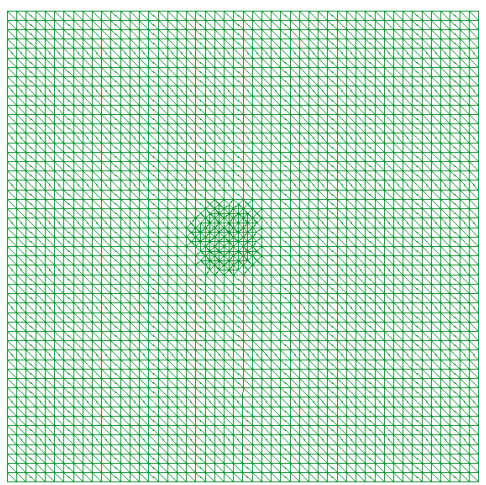

(g) $x y$-projection

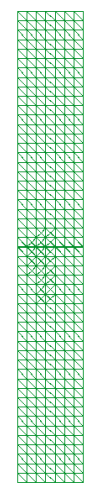

(b) $x z$-projection

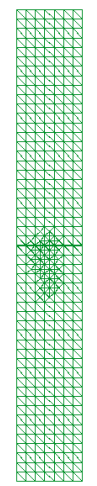

(e) $x z$-projection

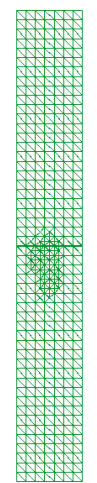

(h) $x z$-projection

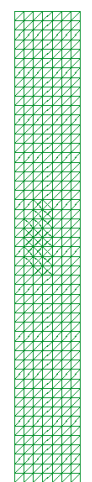

(c) $y z$-projection

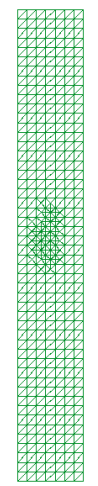

(f) $y z$-projection

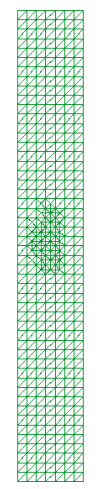

(i) $y z$-projection

Figure 9: Adaptively refined meshes for the target number 3 of Table 1. (a) - (c) once refined, (d) - (f) twice refined, (g) - (i) three times refined mesh. 


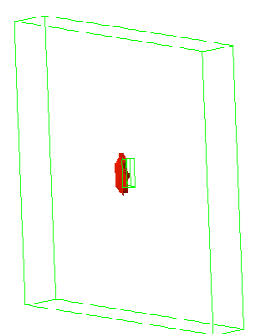

a) coarse mesh

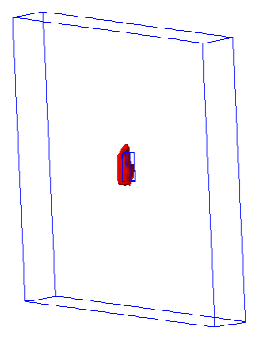

c) once refined

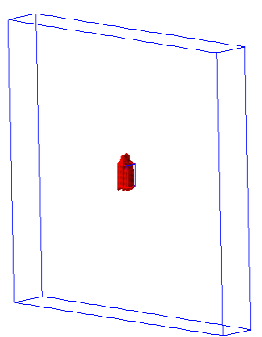

e) twice refined

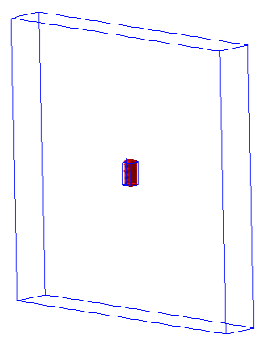

g) three times refined

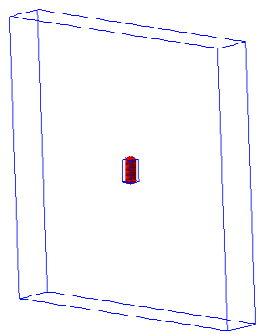

i) four times refined

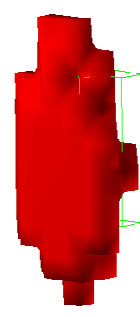

b) zoom

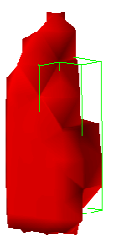

d) zoom

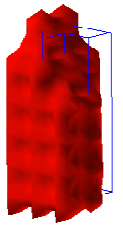

f) zoom

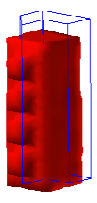

h) zoom

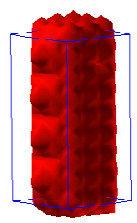

j) zoom

Figure 10: Computed images of target number 4 of Table 1 on four times adaptively refined meshes. Compare with Figure 5 -c). 


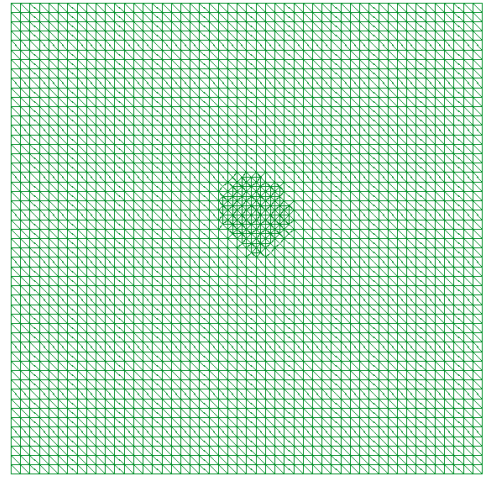

(a)

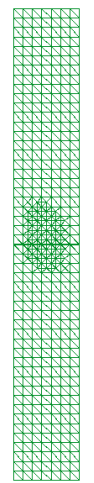

(b)

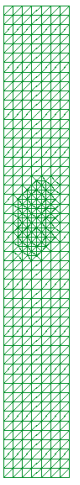

(c)

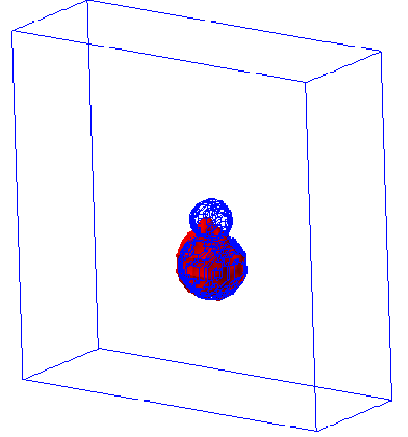

(d)

Figure 11: (a) $x y$-projection, (b) $x z$-projection, and (c) $y z$-projection of the three times refined mesh; d) Computed image of target number 7 (doll, air inside) of Table 1 on that mesh. Thin lines indicate correct shape.

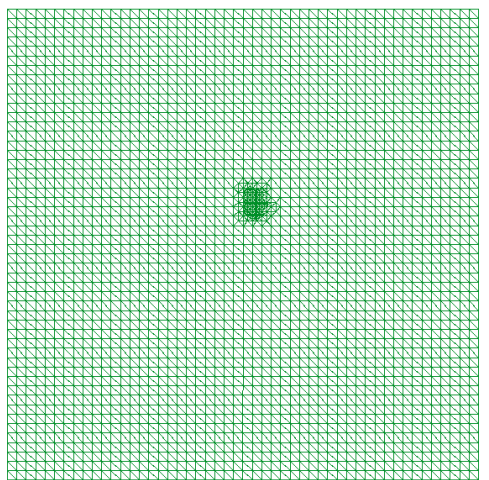

(a)

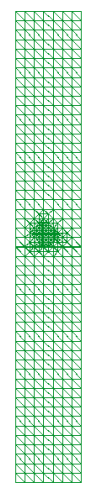

(b)

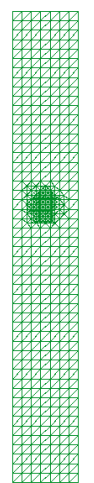

(c)

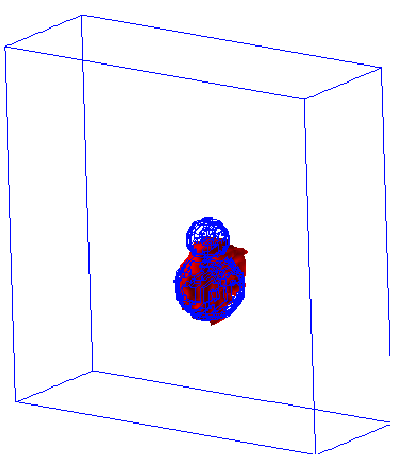

(d)

Figure 12: (a) xy-projection, (b) xz-projection, and (c) yz-projection of the three times refined mesh and the reconstruction $(\mathrm{d})$ of target number 8 on that mesh.

\section{Acknowledgments}

This research was supported by US Army Research Laboratory and US Army Research Office grant W911NF-11-1-0399, the Swedish Research Council, the Swedish Foundation for Strategic Research (SSF) through the Gothenburg Mathematical Modelling Centre (GMMC). The computations were performed on resources at Chalmers Centre for Computational Science and Engineering (C3SE) provided by the Swedish National Infrastructure for Computing (SNIC).

\section{References}

[1] F. Assous, P. Degond, E. Heintze and P. Raviart, On a finite-element method for solving the threedimensional Maxwell equations, J. Comput.Physics, 109, 222-237, 1993. 


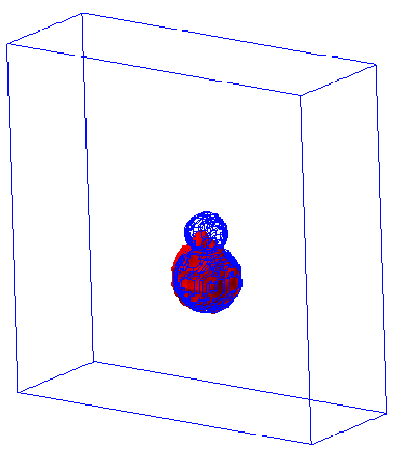

(a) Perspective view

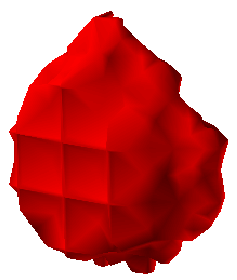

(d) Zoom, perspective

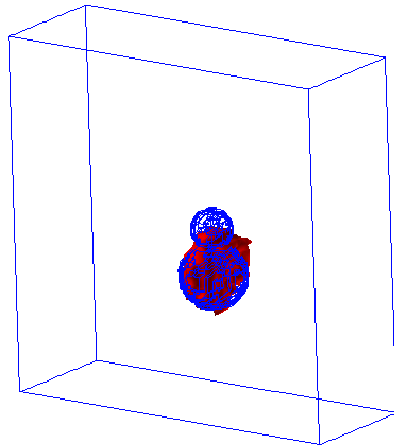

(g) Perspective view

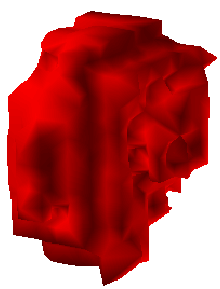

(j) Zoom, perspective

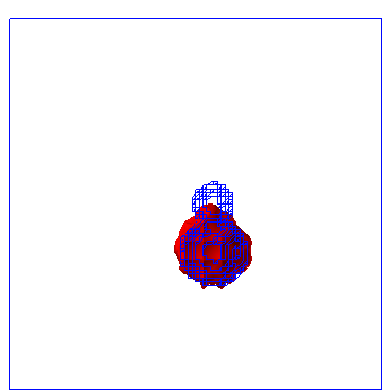

(b) Front view

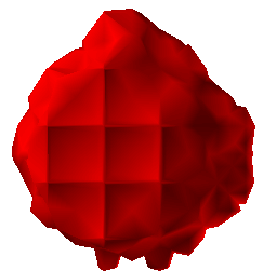

(e) Zoom, front

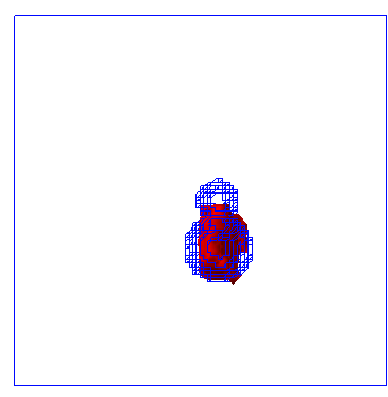

(h) Front view

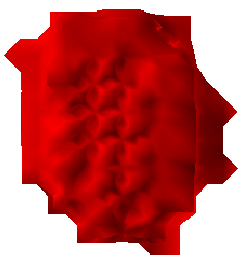

(k) Zoom, front

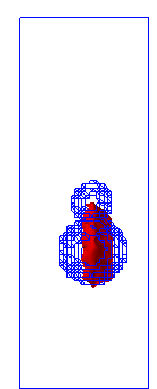

(c) Side view

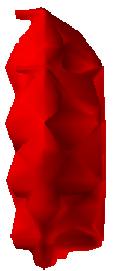

(f) Zoom, side

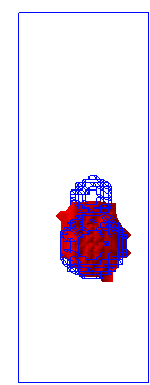

(i) Side view

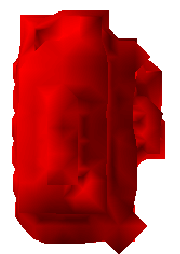

(l) Zoom, side

Figure 13: Three views and zooms of targets number 7 (figures a)-f), doll, air inside) and number 8 (figures g)-1), doll, metal inside) of Table 1 on three times refined mesh. Thin lines indicate correct shape. We observe that on d) and e) even the head of the doll is indicated, which was the most difficult part. As to images g)-1), it follows from (49) that they display mostly the metallic part. Comparison of d), j) with Figures 5 -d), e) again shows a significant improvement of the image due to the adaptivity. 


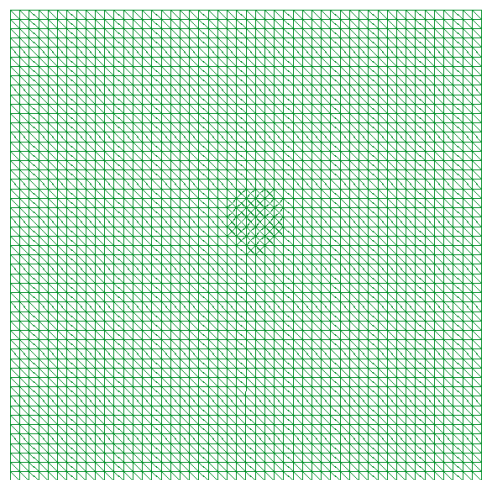

(a) $x y$-projection

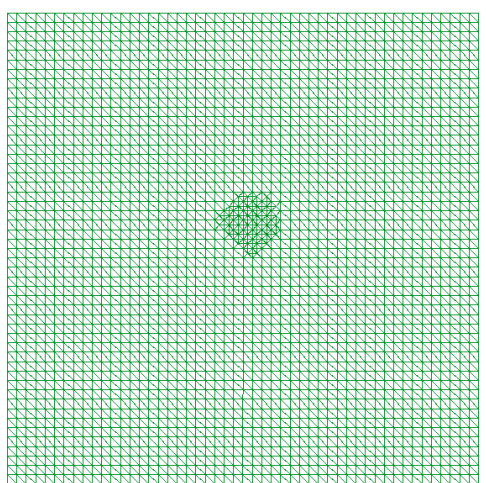

(d) $x y$-projection

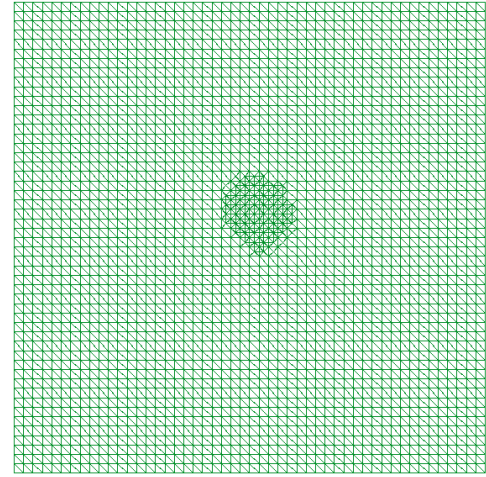

(g) $x y$-projection

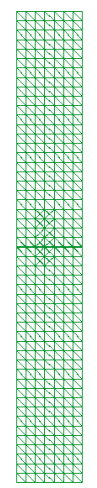

(b) $x z$-projection

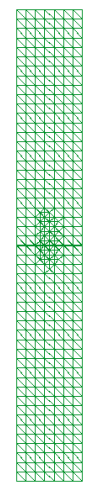

(e) $x z$-projection

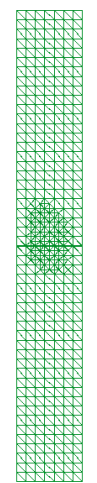

(h) $x z$-projection

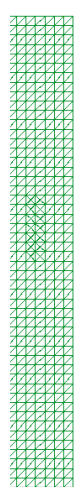

(c) yz-projection

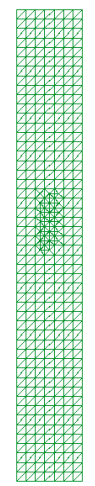

(f) $y z$-projection

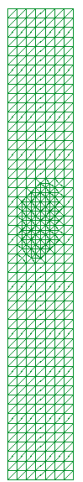

(i) yz-projection

Figure 14: Adaptively refined meshes for target number 7 (doll, air inside) of Table 1 used in our computations. (a) - (c) once refined, (d) - (f) twice refined, (g) - (i) three times refined. 


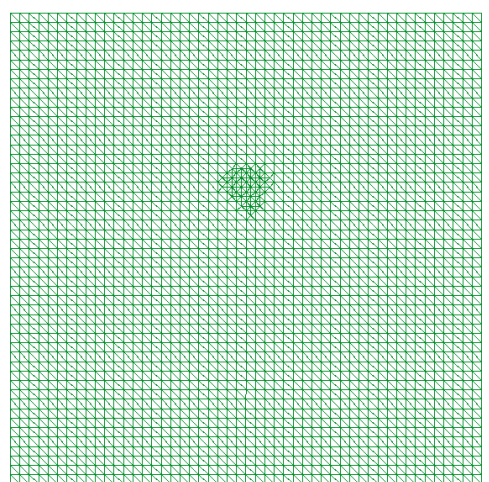

(a)

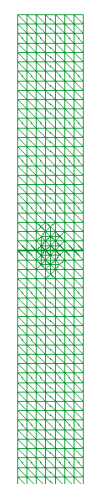

(b)

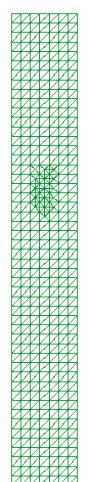

(c)

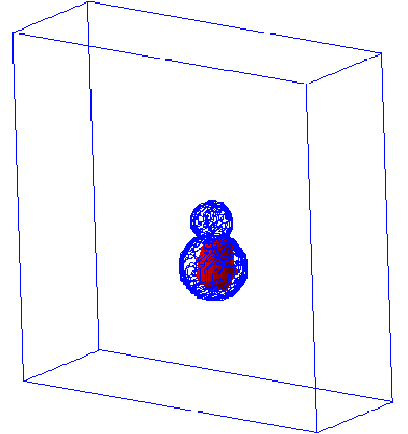

$(d)$

Figure 15: (a) $x y$-projection, (b) $x z$-projection, and (c) yz-projection of the twice refined (optimal) mesh and the reconstruction (d) of target number 9 on that mesh.

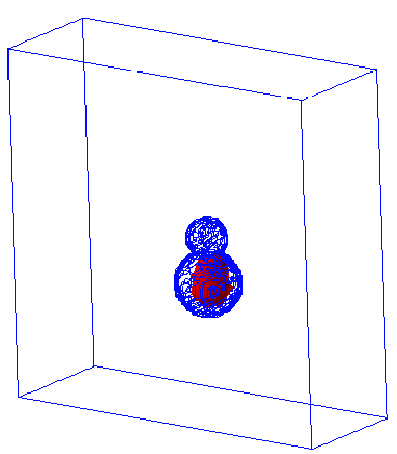

(a) Perspective view

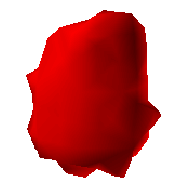

(d) Zoom, perspective

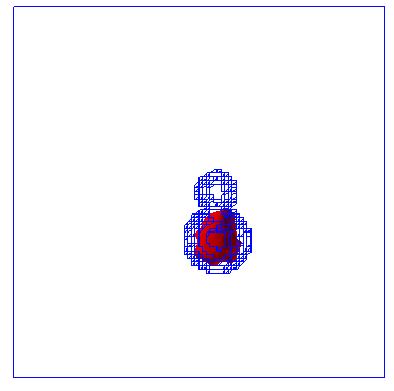

(b) Front view

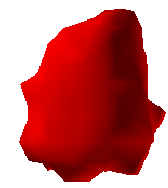

(e) Zoom, front

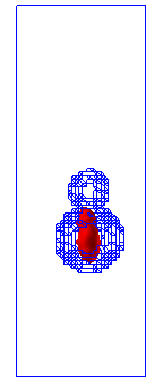

(c) Side view

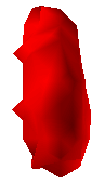

(f) Zoom, side

Figure 16: Three views and zooms of target number 9 of Table 1 on twice refined mesh. Thin lines indicate correct shape. Comparison of d) with Figure 5 f) again shows a significant improvement of the image due to the adaptivity. 
[2] A.B. Bakushinsky and M.Yu. Kokurin, Iterative Methods for Approximate Solutions of Inverse Problems, Springer, New York, 2004.

[3] L. Beilina and M.V. Klibanov, Relaxation property for the adaptivity for ill-posed problems, Applicable Analysis, 93, 223-253, 2014.

[4] L. Beilina and M.V. Klibanov, A globally convergent numerical method for a coefficient inverse problem, SIAM J. Scientific Computing, 31, 478-509, 2008.

[5] L. Beilina and M.V. Klibanov, Synthesis of global convergence and adaptivity for a hyperbolic coefficient inverse problem in 3D, J. Inverse and Ill-Posed Problems, 18, 85-132, 2010.

[6] L. Beilina and M.V. Klibanov, Approximate Global Convergence and Adaptivity for Coefficient Inverse Problems, Springer, New York, 2012.

[7] L. Beilina and M.V. Klibanov, A new approximate mathematical model for global convergence for a coefficient inverse problem with backscattering data, J. Inverse and Ill-Posed Problems, 20, 513-565, 2012.

[8] L. Beilina, Nguyen Trung Thành, M. V. Klibanov and M. A. Fiddy, Reconstruction from blind experimental data for an inverse problem for a hyperbolic equation, Inverse Problems 30, 025002, 2014.

[9] L. Beilina, Energy estimates and numerical verification of the stabilized domain decomposition finite element/finite difference approach for the Maxwell's system in time domain, Central European Journal of Mathematics, 11, 702-733, 2013.

[10] L. Beilina, Adaptive Finite Element Method for a coefficient inverse problem for the Maxwell's system, Applicable Analysis, 90, 1461-1479, 2011.

[11] L. Beilina and C. Johnson, A posteriori error estimation in computational inverse scattering, Mathematical Models in Applied Sciences, 1, 23-35, 2005.

[12] S. C. Brenner and L. R. Scott, The Mathematical theory of finite element methods, Springer-Verlag, Berlin, 1994.

[13] B. Engquist and A. Majda, Absorbing boundary conditions for the numerical simulation of waves, Math. Comp., 31, 629-651, 1977.

[14] V. Isakov, Inverse obstacle problems, Inverse Problems, 25, 123002, 2009.

[15] P. Joly, Variational Methods for Time-Dependent Wave Propagation Problems, Lecture Notes in Computational Science and Engineering, Springer, New York, 2003.

[16] A.V. Kuzhuget, L. Beilina, M.V. Klibanov, A. Sullivan, L. Nguyen and M.A. Fiddy, Blind experimental data collected in the field and an approximately globally convergent inverse algorithm, Inverse Problems, 28, 095007, 2012.

[17] O.A. Ladyzhenskaya, Boundary Value Problems of Mathematical Physics, Springer, New York, 1985.

[18] A. Lakhal, A decoupling based imaging method for inverse medium scattering for Maxwell's equations, Inverse Problems, 26, 015007, 2010.

[19] A. Lakhal, KAIRUAIN-algorithm applied on electromagnetic imaging, Inverse Problems, 29, 095001, 2013.

[20] J. Li, J. Xie and J. Zou, An adaptive finite element reconstruction of distributed fluxes, Inverse Problems, 27, 075009, 2011. 
[21] J. Li, H. Y. Liu, H. Sun and J. Zou, Reconstructing acoustic obstacles by planar and cylindrical waves, J. Math. Phys., 53, 103705, 2012.

[22] J. Li, H. Y. Liu, H. Sun and J. Zou, Imaging acoustic obstacles by singular and hypersingular point sources, Inverse Problems and Imaging, 7, 545-563, 2013.

[23] Y. Liu, J. Su, Z.-J. Lin, S. Teng, A. Rhoden, N. Pantong, and H. Liu. Reconstructions for continuouswave diffuse optical tomography by a globally convergent method. 2013. Preprint, available online at http://www.ma.utexas.edu/mp_arc/, preprint number $13-87$.

[24] O.Pironneau, Optimal shape design for elliptic systems, Springer-Verlag, Berlin, 1984.

[25] R. Stolt, Migration by Fourier transform, Geophysics, 43, 23-48, 1978.

[26] Nguyen Trung Thành, L. Beilina, M. V. Klibanov and M. A. Fiddy, Reconstruction of the refractive index from experimental backscattering data using a globally convergent inverse method, SIAM J. Scientific Computing, accepted for publication; preprint: arXiv:1306.3150 [math-ph], 2013.

[27] N. T. Thành, L. Beilina, M. V. Klibanov, and M. A. Fiddy. Imaging of buried objects from experimental backscattering radar measurements using a globally convergent inverse method. 2014. Submitted.

[28] M. Sini and Nguyen Trung Thành, Inverse acoustic obstacle scattering problems using multifrequency measurements, Inverse Problems and Imaging, 6, 749-773, 2012.

[29] M. Sini and Nguyen Trung Thành, Convergence rates of recursive Newton-type methods for multifrequency scattering, arXiv:1310.5156 [math.NA], 2013.

[30] WavES, the software package, http://www.waves24.com

[31] O. Yilmaz. Seismic Data Imaging. Society of Exploration Geophysicists, Tulsa Oklahoma, 1987. 